\title{
BOUNDARY REGULARITY OF STOCHASTIC PDES
}

\author{
By MÁtÉ GERENCSÉR
}

\begin{abstract}
IST Austria
The boundary behaviour of solutions of stochastic PDEs with Dirichlet boundary conditions can be surprisingly — and in a sense, arbitrarily—bad: as shown by Krylov [SIAM J. Math. Anal. 34 (2003) 1167-1182], for any $\alpha>$ 0 one can find a simple 1-dimensional constant coefficient linear equation whose solution at the boundary is not $\alpha$-Hölder continuous.

We obtain a positive counterpart of this: under some mild regularity assumptions on the coefficients, solutions of semilinear SPDEs on $\mathcal{C}^{1}$ domains are proved to be $\alpha$-Hölder continuous up to the boundary with some $\alpha>0$.
\end{abstract}

1. Introduction. We consider semilinear stochastic partial differential equations (SPDEs) on domains (where the assumptions and precise understanding of the equation is postponed to Section 2) of the type

$$
\begin{aligned}
d u & =\left(a^{i j} D_{i} D_{j} u+f(u, \nabla u)\right) d t+\left(\sigma^{i k} D_{i} u+g^{k}(u)\right) d W_{t}^{k} \quad \text { on } \mathbb{R}_{+} \times G, \\
u & =0 \quad \text { on } \mathbb{R}_{+} \times \partial G, \\
u_{0} & =\psi \quad \text { on } G,
\end{aligned}
$$

with the Einstein summation in place. The well-posedness in the variational sense of a large class of such equations has been known since the 1970s ([18, 24]), and interior regularity (at least for the linear ones) results are available from the 1990s, starting from [10], which initiated a series of works; see, among others, [7, 8, 16, 17, 21, 23]. See also [3] for another approach. Concerning boundary regularity, while the above works give some partial results, the theory is much less satisfactory. Even in the linear case, the rather natural question whether the solution is continuous up to the boundary (and, therefore, whether the boundary condition is actually satisfied in the classical sense) has remained in general unanswered, no matter how smooth the coefficients and the boundary of the domain are, "[becoming] a major challenge for the theory" according to Krylov [9]. Part of the reason why analysing solutions near the boundary is problematic is the fact that the boundary behaviour is indeed quite bad, as illustrated by the following result. Recall that if in the formulation below the coefficient in the noise were greater than $\sqrt{2}$, then the equation would become ill-posed.

Received May 2017; revised March 2018.

MSC2010 subject classifications. 60H15, 35R60.

Key words and phrases. Stochastic partial differential equations, Dirichlet problem, boundary regularity. 
THEOREM 1.1 ([13]). There exists a $\lambda_{0}>0$ such that if $0<\lambda<\lambda_{0}, \psi \in$ $\mathcal{C}_{0}^{\infty}\left(\mathbb{R}_{+}\right)$is nonidentically 0 , and $u$ denotes the solution of

$$
\begin{aligned}
d u & =D^{2} u d t+\sqrt{2-\lambda} D u d W_{t} \quad \text { on } \mathbb{R}_{+} \times \mathbb{R}_{+}, \\
u & =0 \quad \text { on } \mathbb{R}_{+} \times\{0\}, \\
u_{0} & =\psi \quad \text { on } \mathbb{R}_{+},
\end{aligned}
$$

then almost surely there exists a dense subset $S \subset \mathbb{R}_{+}$such that for all $s \in S$ and $\alpha>e^{-\frac{1}{2 \lambda}}$

$$
\lim _{x \downarrow 0} u_{s}(x) x^{-\alpha}=\infty
$$

The main goal of the present article is to prove that solutions of (1) are Höldercontinuous up to the boundary, with some exponent. In light of the above, this exponent of course has to depend on the equation itself, and as we will see, this dependence is in fact only on through a few parameters of the linear part of the equation. Since the precise statement requires a bit of technical setup, we postpone it to the next section; see Theorem 2.6. Our proof is inspired by [14], where the particular case of $d=1, f=g=\nabla a=\nabla \sigma=0$, was treated. Importantly, unlike the above mentioned "partial" results, its approach relied neither on a "smallness" nor on a "compatibility" condition on $\sigma$.

To our best knowledge, the most general well-posedness results for (1) use the variational theory, which however strongly restricts the growth of $f$. We prove a more general existence and uniqueness result in Theorem 3.2. That itself requires no growth assumption at all on $f(u, \nabla u)$ in $u$, and this allows us to state also Theorem 2.6 under mild (arbitrary polynomial) growth conditions.

The article is organised as follows. In the following section, after setting up most of the notation, the main result is stated, which is followed by the aforementioned solvability result in Section 3, and the rest of the paper is devoted to the proof of Theorem 2.6. The proof has four main components: reducing the problem to equations with linear structure and more regular data, transforming the simplified equation to a PDE with random coefficients on a random domain, establishing certain geometric properties of this random domain and finally using these properties to prove the appropriate decay at the boundary. Section 4 is structured according to these steps.

2. Formulation. Fix a complete filtered probability space $\left(\Omega,\left(\mathcal{F}_{t}\right)_{t \geq 0}, P\right)$ carrying an infinite sequence of independent Wiener processes $\left(W_{t}^{k}\right)_{k \in \mathbb{N}, t \geq 0}$. The predictable $\sigma$-algebra on $\Omega \times \mathbb{R}_{+}$is denoted by $\mathcal{P}$. Whenever expectations are taken with respect to a different probability measure $\hat{P}$, it will be denoted by $\mathbb{E}^{\hat{P}}$. Let us also fix $T>0$. Given a $d$-dimensional stochastic differential equation (SDE),

$$
d X_{t}^{i}=\alpha^{i}\left(X_{t}\right) d t+\beta^{i k}\left(X_{t}\right) d W_{t}^{k}, \quad i=1,2, \ldots, d
$$


driven by $W$, the corresponding stochastic flow on $[0, T]$ is a continuous random field $\left(X_{s, t}(x)\right)_{0 \leq s \leq t \leq T, x \in \mathbb{R}^{d}}$ such that for all $s$ and $x$, the process $\left(X_{s, t}(x)\right)_{s \leq t \leq T}$ is a solution of equation (2) with initial condition $X_{s, s}(x)=x$, and that furthermore almost surely for all $0 \leq s \leq t \leq v \leq T$ and $x \in \mathbb{R}^{d}$, the identity $X_{t, v}\left(X_{s, t}(x)\right)=$ $X_{s, v}(x)$ holds. When the stochastic differential in (2) is replaced by the backward Itô differential $d \overleftarrow{W}_{t}$, then one can correspondingly talk about the backward flow $\left(X_{t, s}(x)\right)_{0 \leq s \leq t \leq T, x \in \mathbb{R}^{d}}$. Often it turns out that for any $0 \leq s \leq t \leq T, X_{s, t}(\cdot)$ is a diffeomorphism from $\mathbb{R}^{d}$ to itself, in which case one can talk about the inverse flow $\left(X_{s, t}^{-1}(x)\right)_{0 \leq s \leq t \leq T, x \in \mathbb{R}^{d}}$.

By $B_{r}(x)$, we understand the $d$-dimensional ball of radius $r \geq 0$ around $x \in \mathbb{R}^{d}$, and for $x=0$ the $x$ argument is often dropped. We denote by $\langle\cdot, \cdot\rangle$ the scalar product in $\mathbb{R}^{d}$. The distance between two closed sets $A$ and $B$ is denoted by $d(A, B)$. The Borel $\sigma$-algebra on $\mathbb{R}^{n}$ is denoted by $\mathcal{B}\left(\mathbb{R}^{n}\right)$.

We fix a bounded $\mathcal{C}^{1}$-domain $G \subset \mathbb{R}^{d}$ (as defined in, e.g., [8]), denote $G^{c}=\mathbb{R}^{d} \backslash$ $G, Q=[0, T] \times G, G^{+}=G+B_{1}:=\left\{x \in \mathbb{R}^{d}: \exists x_{1} \in G, x_{2} \in B_{1}: x=x_{1}+x_{2}\right\}$, $Q^{+}=[0, T] \times G^{+}$and for $T_{0} \geq 0, Q_{T_{0}}=\left[T_{0}, T\right] \times G$. Fix a $\mathcal{C}^{\infty}$ function $\Psi$ defined on $G$ such that for all $x \in G$,

$$
d(x, \partial G) \leq N \Psi(x) \leq N^{\prime} d(x, \partial G), \quad d(x, \partial G)^{|k|}\left|D_{k} \nabla \Psi(x)\right| \leq N(k)
$$

for some constants $N, N^{\prime},(N(k)), k$ running over all possible multiindices. For the existence of such function see, for example, [22].

Derivatives in the direction of the $i$ th unit direction in $\mathbb{R}^{d}$ are denoted by $D_{i}$. By $\nabla$, we denote the gradient, with the convention that for $f: \mathbb{R}^{d} \rightarrow \mathbb{R}^{k},(\nabla f)^{i j}=$ $D_{j} f^{i}$.

For $\gamma \in \mathbb{R}$ and $p \geq 1$, by $H_{p}^{\gamma}=H_{p}^{\gamma}(G)$ we mean the usual Sobolev spaces; see, for example, [26]. By $\dot{H}_{p}^{\gamma}$, we mean the closure of $\mathcal{C}_{0}^{\infty}(G)$ in the $H_{p}^{\gamma}$ norm. For $\gamma, \theta \in \mathbb{R}$ and $p \geq 1$, by $H_{p, \theta}^{\gamma}=H_{p, \theta}^{\gamma}(G)$ we understand weighted Sobolev spaces. An easily accessible definition of them is to first set for $\gamma=n \in \mathbb{N}$,

$$
\|u\|_{H_{p, \theta}^{\gamma}}^{p}:=\sum_{i=0}^{n} \sum_{|\alpha|=i} \int_{G}\left|D_{\alpha_{1}} \cdots D_{\alpha_{i}} u\right|^{p}(x) d(x, \partial G)^{\theta-d+i p} d x,
$$

and then extend this scale of spaces to noninteger and nonnegative values of $\gamma$ by interpolation and duality, respectively; see [22] and [11] for more details, and also for a more intrinsic equivalent definition of these spaces.

Hölder spaces $\mathcal{C}^{\alpha}(A)$ on some set $A \subset \mathbb{R}^{n}$ for $\alpha \in(0,1]$ are defined with the norm

$$
\|u\|_{\mathcal{C}^{\alpha}(A)}:=\sup _{x \in A}|u(x)|+\sup _{x \neq y \in A} \frac{|u(x)-u(y)|}{|x-y|^{\alpha}} .
$$

For $\alpha>0, u \in \mathcal{C}^{\alpha}$ if all of its $k$ th derivatives, $|k|<\lceil\alpha\rceil$, belong to $\mathcal{C}^{\alpha+1-\lceil\alpha\rceil}$.

All of the above spaces can easily be extended to $l_{2}$-valued (or $\left(l_{2}\right)^{n}$-valued, for that matter) functions, by taking the appropriate operations coordinatewise and 
replace the absolute value by the $l_{2}$-norm. Hence the dimension of the function spaces will not always be detailed; for example, the reader understands that requiring the coefficient $\beta$ of an equation like (2) to be of class $\mathcal{C}^{1}$ is to require it to be an element of $\mathcal{C}^{1}\left(\mathbb{R}^{d},\left(l_{2}\right)^{d}\right)$.

The understanding of the solution of (1) is the following.

DEFINITION 2.1. A solution of (1) is a continuous adapted $L_{2}$-valued process $u$ that furthermore belongs to $L_{\infty}(Q) \cap L_{2}\left([0, T], \dot{H}_{2}^{1}(G)\right)$ almost surely, such that for all $\varphi \in \mathcal{C}_{0}^{\infty}(G)$ the identity

$$
\begin{aligned}
\left(u_{t}, \varphi\right)= & (\psi, \varphi) \\
& +\int_{0}^{t}\left(-D_{j} u_{s}, a_{s}^{i j} D_{i} \varphi\right)+\left(f_{s}\left(u_{s}, \nabla u_{s}\right)-\left(D_{i} a_{s}^{i j}\right) D_{j} u_{s}, \varphi\right) d s \\
& +\int_{0}^{t}\left(\sigma_{s}^{i k} D_{i} u_{s}+g_{s}^{k}\left(u_{s}\right), \varphi\right) d W_{s}^{k}
\end{aligned}
$$

holds almost surely for all $t \in[0, T]$, where $(\cdot, \cdot)$ denotes the $L_{2}$-inner product.

Our assumptions for the main result are as follows (in particular, they are more than sufficient to guarantee that all expressions in (4) make sense).

Assumption 2.2. There exists a $\kappa>0$ such that for all $(t, \omega, x) \in[0, T] \times$ $\Omega \times\left(G+B_{1 / 2}\right)$,

$$
\bar{a}:=a-\frac{1}{2} \sigma \sigma^{*} \geq \kappa I
$$

holds in the sense of positive semidefinite matrices.

AsSUMPTION 2.3. (a) The coefficients $a$ and $\sigma$ are $\mathcal{P} \otimes \mathcal{B}\left(\mathbb{R}^{d}\right)$-measurable functions that vanish outside $G^{+}$. There exist constants $K>0$ and $v \in(0,1)$ such that for all $t$ and $\omega$,

$$
\left\|a_{t}(\cdot)(\omega)\right\|_{\mathcal{C}^{2+v}\left(\mathbb{R}^{d}\right)}+\left\|\sigma_{t}(\cdot)(\omega)\right\|_{\mathcal{C}^{3+v}\left(\mathbb{R}^{d}\right)} \leq K .
$$

(b) There exists a random variable $H$ with finite moments of all order such that for all $\omega$,

$$
\|\sigma .(\cdot)(\omega)\|_{\mathcal{C}^{v}\left([0, T], L_{\infty}\left(\mathbb{R}^{d}\right)\right)} \leq H(\omega)
$$

Assumption 2.4. (a) The function $f(u, \nabla u)$ takes the form $f(u, \nabla u)=$ $\bar{f}(u)+\nabla \cdot(\hat{f}(u))$, with $\hat{f}(0)=0$. The functions $\bar{f}, \hat{f}$ and $g$ are $\mathcal{P} \otimes \mathcal{B}\left(\mathbb{R}^{d}\right) \otimes$ $\mathcal{B}(\mathbb{R})$-measurable, with values in $\mathbb{R}, \mathbb{R}^{d}$ and $l_{2}$, respectively, that vanish outside 
$G^{+}$. The real-valued function $\psi$ is $\mathcal{F}_{0} \otimes \mathcal{B}\left(\mathbb{R}^{d}\right)$-measurable and vanishes outside $G^{+}$. The function $\bar{f}_{t}(x, y)(\omega)$ is continuous in $y \in \mathbb{R}$ uniformly in $t, x, \omega$ and there exists a constant $K>0$ such that

$$
\begin{aligned}
\left(y-y^{\prime}\right) & \left(\bar{f}_{t}(x, y)(\omega)-\bar{f}_{t}\left(x, y^{\prime}\right)(\omega)\right) \leq K\left|y-y^{\prime}\right|^{2}, \\
\left|\hat{f}_{t}(x, y)(\omega)-\hat{f}_{t}\left(x, y^{\prime}\right)(\omega)\right| & \leq K\left|y-y^{\prime}\right|, \\
\left|g_{t}(x, y)(\omega)-g_{t}\left(x, y^{\prime}\right)(\omega)\right| & \leq K\left|y-y^{\prime}\right|
\end{aligned}
$$

for all $t, x, y, y^{\prime}, \omega$.

(b) There exists a constant $m>0$ such that for all $t, x, y, \omega$,

$$
\left|\bar{f}_{t}(x, y)(\omega)-\bar{f}_{t}(x, 0)(\omega)\right| \leq K|y|^{m} .
$$

Assumption 2.5. The functions $\psi, f^{0}=f_{t}^{0}(x):=\bar{f}_{t}(x, 0)$ and $g^{0}:=$ $g_{t}(x)=g_{t}(x, 0)$ satisfy, for some $\bar{v}>0$ and for all $p \in[2, \infty)$

$$
\begin{aligned}
& \mathbb{E}\left(\|\psi\|_{H_{p}^{\bar{v}}}+\left\|f^{0}\right\|_{L_{d+4}\left([0, T], H_{d+4}^{-1+\bar{\nu}}\right)}+\left\|f^{0}\right\|_{L_{p}\left([0, T], H_{p, d-2+2 p}^{-2+\bar{v}}\right)}\right. \\
& \left.+\left\|g^{0}\right\|_{L_{d+4}\left([0, T], H_{d+4, d-1 / 2}^{\bar{\nu}}\right)}+\left\|g^{0}\right\|_{L_{p}\left([0, T], H_{p, d-2+p}^{-1+\bar{v}}\right)}\right)^{2}<\infty .
\end{aligned}
$$

Let us finally denote $d_{1}:=\inf \left\{k \in \mathbb{N}: \sigma_{t}^{i l}(x)(\omega) \equiv 0 \forall l>k\right\}$.

These assumptions, unless one assumes further control of the growth of $\bar{f}$ in $u$, are not quite enough to fit in the $L_{2}$-theory $([18,24])$, and in fact as far as the author is aware, no result on well-posedness in this scope is known. In the next section, we prove some existence and uniqueness results that well cover the above setting. The main result of the paper then reads as follows.

THEOREM 2.6. Let Assumptions 2.2 and 2.3 hold and suppose $d_{1}<\infty$. Then there exists an $\alpha=\alpha\left(\kappa, K, \bar{v}, d, d_{1}\right)>0$ such that for any $T_{0}>0$ and $\psi, f, g$ satisfying Assumptions 2.4 and 2.5, a unique solution $u$ of (1) exists and almost surely

$$
\sup _{(t, x) \in Q_{T_{0}}}|u(t, x)| d(x, \partial G)^{-\alpha}<\infty .
$$

Moreover, for fixed $K, \bar{v}, d, d_{1}$, there exists a $c_{0}$ such that for sufficiently small $\kappa$ one has $\alpha>e^{-c_{0} / \kappa}$.

REMARK 2.7. Since $\psi$ is not assumed to vanish at the boundary, one can in general not take $T_{0}=0$. Concerning the assumption $d_{1}<\infty$, one could actually do slightly better with essentially the same argument; see Remark 4.13 below.

REMARK 2.8. Assumption 2.5 is somewhat cumbersome. A stronger, but perhaps more tractable condition would be

$$
\mathbb{E}\left(\|\psi\|_{H_{\tilde{\tilde{p}}}^{\tilde{\tilde{v}}}}+\left\|f^{0}\right\|_{L_{\infty}\left([0, T], H_{\tilde{p}}^{-1+\tilde{\nu}}\right)}+\left\|\Psi^{-1 /(2(d+4))} g^{0}\right\|_{L_{\infty}\left([0, T], H_{\tilde{\tilde{p}}}^{\tilde{\tilde{p}}}\right)}\right)^{2}<\infty
$$


with some fixed $\tilde{v}>0, \tilde{p}>d / \tilde{v}$. As one can see from the basic properties of weighted Sobolev spaces (which we recall in Section 4.1), (5) implies Assumption 2.5 , with $\bar{v}=\tilde{v}-d / \tilde{p}$. One reason why one would not want to impose (5), however, is that it assumes some pointwise decay at the boundary from $g^{0}$, while Assumption 2.5 does not.

Combining Theorem 2.6 and some interior regularity, one easily gets the following corollary, which is proved in Section 4.1 .

COROLlary 2.9. Assume the setting of Theorem 2.6 and let $\hat{\alpha}$ satisfy

$$
0<\hat{\alpha}<\frac{\alpha \bar{v}}{3(\alpha+\bar{v})} .
$$

Then for any $T_{0}>0$, the solution $u$ of (1) belongs to $\mathcal{C}^{\hat{\alpha}}\left(\overline{Q_{T_{0}}}\right)$ almost surely.

3. Existence and uniqueness of the solution. First we state the existence result under some reduced regularity and growth assumptions. Note that we momentarily switch to equations in divergence form, but since in the rest of the article the regularity condition Assumption 2.3 on the coefficients will be in place, switching between divergence and nondivergence form equations is harmless. We also remark that for Theorem 3.2 one in fact only needs $G$ to be a Lipschitz domain.

Assumption 3.1. The functions $\psi, f^{0}$, and $g^{0}$ satisfy, for some $\mu>0$,

$$
\mathcal{K}_{0}:=\|\psi\|_{L_{\infty}(G)}+\left\|f^{0}\right\|_{L_{d+2+\mu}\left([0, T], H_{d+2+\mu}^{-1}\right)}+\left\|g^{0}\right\|_{L_{d+2+\mu}(Q)}<\infty
$$

almost surely.

Define also

$$
\mathcal{K}_{1}:=\|\psi\|_{L_{2}(G)}+\left\|f^{0}\right\|_{L_{2}\left([0, T], H_{2}^{-1}\right)}+\left\|g^{0}\right\|_{L_{2}(Q)} .
$$

THEOREM 3.2. Let Assumptions 2.2, 2.4(a) and 3.1 hold and assume that $a$ and $\sigma$ are $\mathcal{P} \otimes \mathcal{B}\left(\mathbb{R}^{d}\right)$-measurable functions bounded by $K$. Then there exists a unique continuous $L_{2}$-valued adapted process $u$ that furthermore belongs to $L_{\infty}(Q) \cap L_{2}\left([0, T], \dot{H}_{2}^{1}(G)\right)$ such that for all $\varphi \in \mathcal{C}_{0}^{\infty}(G)$ the identity

$$
\begin{aligned}
\left(u_{t}, \varphi\right)= & (\psi, \varphi)+\int_{0}^{t}\left(-D_{j} u_{s}, a_{s}^{i j} D_{i} \varphi\right)+\left(f_{s}\left(u_{s}, \nabla u_{s}\right), \varphi\right) d s \\
& +\int_{0}^{t}\left(\sigma^{i k} D_{i} u_{s}+g_{s}^{k}\left(u_{s}\right), \varphi\right) d W_{s}^{k}
\end{aligned}
$$

holds almost surely for all $t \in[0, T]$. Finally, the estimates

$$
\begin{aligned}
\mathbb{E}\|u\|_{L_{\infty}(Q)}^{p} & \leq N(\kappa, K, \mu, T, d, G, p) \mathbb{E}_{0}^{p}, \\
\mathbb{E}\|u\|_{L_{2}\left([0, T], H_{2}^{1}(G)\right)}^{2} & \leq N(\kappa, K, T, d, G) \mathbb{E} \mathcal{K}_{1}^{2}
\end{aligned}
$$

hold with any $p \in(0, \infty)$. 
PROOF. The proof closely follows those of Theorems 2.1-5.2 in [1] and (as, in fact, indicated therein) one needs only make sure that the nonlinear terms do not change anything essential. We therefore do not aim to repeat the whole argument, but rather will only detail the verification of this.

Define for $n, m \in \mathbb{N}$, $\bar{f}_{t}^{(n, m)}(x, y):=\bar{f}_{t}(x,-n \vee y \wedge m), \quad f^{(n, m)}(u, \nabla u)=\bar{f}^{(n, m)}(u)+\nabla \cdot(\hat{f}(u))$. Since $\bar{f}^{(n, m)}$ has linear growth, the results of [18] apply, and hence one has the existence of a unique continuous $L_{2}$ valued adapted process $u^{(n, m)}$ which furthermore belongs to $L_{2}\left([0, T], \dot{H}_{2}^{1}(G)\right)$ and such that (6) holds with $u^{(n, m)}$ and $f^{(n, m)}$ in place of $u$ and $f$, respectively.

Applying Itô's formula ([1], Lemma 3.2) to $\left\|u_{t}^{(n, m)}\right\|_{L_{p}(G)}^{p}, p \geq 2$, one gets

$$
\begin{aligned}
\int_{G}\left|u_{t}^{(n, m)}\right|^{p} d x \\
=\int_{G}|\psi|^{p} d x \\
\quad+\int_{0}^{t} \int_{G} p\left|u_{s}^{(n, m)}\right|^{p-2} u_{s}^{(n, m)}\left(\sigma_{s}^{i k} D_{i} u_{s}^{(n, m)}+g_{s}^{k}\left(u_{s}^{(n, m)}\right)\right) d x d W_{s}^{k} \\
\quad+\int_{0}^{t} \int_{G}-p(p-1)\left|u_{s}^{(n, m)}\right|^{p-2} D_{i} u_{s}^{(n, m)} a_{s}^{i j} D_{j} u_{s}^{(n, m)} \\
\quad+p\left|u_{s}^{(n, m)}\right|^{p-2} u_{s}^{(n, m)} \bar{f}_{s}^{(n, m)}\left(u_{s}^{(n, m)}\right) \\
\quad-p(p-1)\left|u_{s}^{(n, m)}\right|^{p-2} \nabla u_{s}^{(n, m)} \cdot \hat{f}_{s}^{(n, m)}\left(u_{s}^{(n, m)}\right) \\
\quad+(1 / 2) p(p-1)\left|u_{s}^{(n, m)}\right|^{p-2}\left|\sigma_{s}^{i k} D_{i} u_{s}^{(n, m)}+g_{s}^{k}\left(u_{s}^{(n, m)}\right)\right|_{l_{2}}^{2} d x d s .
\end{aligned}
$$

Looking at the contribution of the nonlinear terms, we can write, by Assumption 2.4(a)

$$
u_{s}^{(n, m)} \bar{f}_{s}^{(n, m)}\left(u_{s}^{(n, m)}\right) \leq K\left|u_{s}^{(n, m)}\right|^{2}+u_{s}^{(n, m)} f_{s}^{0} .
$$

Recall that by Assumption 3.1, $f^{0}=\bar{h}^{0}+\nabla \cdot \hat{h}^{0}$, where $\bar{h}^{0}, \hat{h}^{0} \in L_{d+2+\mu}(Q)$

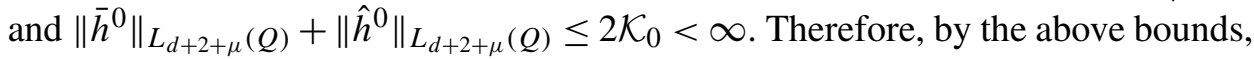
integration by parts, and Young's inequality we have, for any $\varepsilon>0$,

$$
\begin{aligned}
\int_{G} p \mid & \left.u_{s}^{(n, m)}\right|^{p-2} u_{s}^{(n, m)} \bar{f}_{s}^{(n, m)}\left(u_{s}^{(n, m)}\right) d x \\
\leq & \int_{G} p^{2}\left|u_{s}^{(n, m)}\right|^{p-2} \\
& \quad \times\left(K\left|u_{s}^{(n, m)}\right|^{2}+\varepsilon\left|\nabla u_{s}^{(n, m)}\right|^{2}+\left|u_{s}^{(n, m)}\right|\left|\bar{h}_{s}^{0}\right|+C(\varepsilon)\left|\hat{h}_{s}^{0}\right|^{2}\right) d x
\end{aligned}
$$

for some constant $C(\varepsilon)$ depending only on $\varepsilon$ and $K$. Next, we have

$$
\left|\nabla u_{s}^{(n, m)} \cdot \hat{f}_{s}^{(n, m)}\left(u_{s}^{(n, m)}\right)\right| \leq \varepsilon\left|\nabla u_{s}^{(n, m)}\right|^{2}+C(\varepsilon)\left|u_{s}^{(n, m)}\right|^{2},
$$


allowing one to bound the second to last term in (7) As for the contribution of $g$, one simply has

$$
\begin{aligned}
\left(2 \sigma_{s}^{i k} D_{i} u_{s}^{(n, m)}+g_{s}^{k}\left(u_{s}^{(n, m)}\right)\right) g_{s}^{k}\left(u_{s}^{(n, m)}\right) & \\
= & \left(2 \sigma_{s}^{i k} D_{i} u_{s}^{(n, m)}\right. \\
\quad & \left.\quad\left(g_{s}^{k}\left(u_{s}^{(n, m)}\right)-\left(g_{s}^{0}\right)^{k}\right)+\left(g_{s}^{0}\right)^{k}\right)\left(\left(g_{s}^{k}\left(u_{s}^{(n, m)}\right)-\left(g_{s}^{0}\right)^{k}\right)+\left(g_{s}^{0}\right)^{k}\right) .
\end{aligned}
$$

Therefore, by Assumption 2.4(a) we have, for any $\varepsilon>0$,

$$
\begin{aligned}
& \sum_{k \geq 0} \int_{G}(1 / 2) p(p-1)\left|u_{s}^{(n, m)}\right|^{p-2}\left(2 \sigma_{s}^{i k} D_{i} u_{s}^{(n, m)}+g_{s}^{k}\left(u_{s}^{(n, m)}\right)\right) g_{s}^{k}\left(u_{s}^{(n, m)}\right) d x \\
& \quad \leq p^{2} \int_{G} C(\varepsilon)\left|u_{s}^{(n, m)}\right|^{p}+C(\varepsilon)\left|u_{s}^{(n, m)}\right|^{p-2}\left|g^{0}\right|^{2}+\varepsilon\left|u_{s}^{(n, m)}\right|^{2}\left|\nabla u_{s}^{(n, m)}\right|^{2} d x .
\end{aligned}
$$

All of these are of precisely the same order as the contributions coming from the lower order linear terms in [1]. Note also that the constants on the right-hand sides do not depend on $n$ and $m$. The resulting energy estimates are therefore virtually identical to the ones in the linear case, and thus so is Moser's iteration. One therefore obtains the bounds

$$
\mathbb{E}\left\|u^{(n, m)}\right\|_{L_{\infty}(Q)}^{p} \leq N \mathbb{E} \mathcal{K}_{0}^{p}
$$

with $N$ depending on $\kappa, K, \mu, T, d, G, p$ but not on $n$ and $m$. Also, applying Itô's formula for $\left\|u_{s}\right\|_{L_{2}(G)}^{2}$, by the above and Assumption 2.2 one gets

$$
\begin{aligned}
\int_{G}\left|u_{T}^{(n, m)}\right|^{2} d x \leq & \int_{G}|\psi|^{2} d x+M_{T}-2 \kappa \int_{0}^{T} \int_{G}\left|\nabla u_{s}^{(n, m)}\right|^{2} d x d s \\
& +\int_{0}^{T} \int_{G} C(\varepsilon)\left(\left|u_{s}^{(n, m)}\right|^{2}+\left|\bar{h}_{s}^{0}\right|^{2}+\left|\hat{h}_{s}^{0}\right|^{2}+\left|g_{s}^{0}\right|^{2}\right) \\
& +\varepsilon\left|\nabla u_{s}^{(n, m)}\right|^{2} d x d s
\end{aligned}
$$

with some martingale $M$. Hence one obtains

$$
\mathbb{E}\left\|u_{s}^{(n, m)}\right\|_{L_{2}\left([0, T], H_{2}^{1}(G)\right)}^{2} \leq N \mathbb{E} \mathcal{K}_{1}^{2}
$$

with $N$ having the same dependencies as before, except for $\mu$ and $p$.

Now we let $n \rightarrow \infty$. By the comparison principle [2], Theorem 3.3, one has that $u^{(n, m)} \leq u^{\left(n^{\prime}, m\right)}$ for $n^{\prime} \geq n$, which, thanks to (8), implies that $u^{(n, m)}$ not only converges as $n \rightarrow \infty$, but is in fact constant in $n$ after an index $N=N(\omega)$. This implies that the limit $u^{(\infty, m)}$ is a solution of (6) with $f$ replaced by

$$
f^{(\infty, m)}(u, \nabla u)=\bar{f}^{(\infty, m)}(u)+\nabla \cdot(\hat{f}(u)), \quad \bar{f}_{t}^{(\infty, m)}(x, y):=\bar{f}_{t}(x, y \wedge m),
$$

and moreover, $u_{s}^{(\infty, m)}$ also satisfies the bounds (8)-(9). One then passes to the $m \rightarrow \infty$ limit similarly, and the limit $u:=u^{(\infty, \infty)}$ is indeed the solution claimed in the theorem. 
As for the uniqueness, take two solutions $u$ and $v$ and write Itô's formula for $\|e\|_{L_{2}(G)}^{2}:=\|u-v\|_{L_{2}(G)}^{2}$ :

$$
\begin{aligned}
\int_{G}\left|e_{t}\right|^{2} d x= & \int_{0}^{t} \int_{G}-\overline{2} a_{s}^{i j} D_{i} e_{s} D_{j} e_{s}+\left(u_{s}-v_{s}\right)\left(f_{s}\left(u_{s}, \nabla u_{s}\right)-f_{s}\left(v_{s}, \nabla v_{s}\right)\right) \\
& +2 \sigma_{s}^{i k} D_{i}\left(u_{s}-v_{s}\right)\left(g_{s}^{k}\left(u_{s}\right)-g_{s}^{k}\left(v_{s}\right)\right)+\left|g_{s}\left(u_{s}\right)-g_{s}\left(v_{s}\right)\right|^{2} d x d s \\
& +m_{t}
\end{aligned}
$$

with some martingale $m$. By Assumption 2.4(a), one has

$$
(u-v)(f(u, \nabla u)-f(v, \nabla v)) \leq K|u-v|^{2}+(u-v) \nabla(\hat{f}(u)-\hat{f}(v)) .
$$

After integration by parts, using simply the bound $|g(u)-g(v)| \leq K|u-v|$ in the terms involving $g$, and by Assumption 2.2, we get

$$
\int_{G}\left|e_{t}\right|^{2} \leq \int_{0}^{t} \int_{G}-2 \kappa\left|\nabla e_{s}\right|^{2}+C\left|e_{s}\right|^{2}+C^{\prime}\left|e_{s}\right|\left|\nabla e_{s}\right| d x d s+m_{t}
$$

with some constants $C, C^{\prime}$ depending on $K$. Hence, Young's inequality, taking expectations, and Gronwall's lemma yields $\left(\mathbb{E}\left\|e_{t}\right\|_{L_{2}(G)}^{2}\right)_{t \in[0, T]} \equiv 0$. Since $e$ is continuous in $L_{2}(G),\left(e_{t}\right)_{t \in[0, T]} \equiv 0$ almost surely, as required.

\section{Proof of Theorem 2.6.}

4.1. Simplifying. As a first step, we reduce the statement to a version where the equation is linear, $f$ is regular and $g$ is simply not present. To do that, however, we need to derive some further properties of the solution of (1), based on $L_{p^{-}}$ theory, and so we recall some notation from it.

We somewhat deviate from the standard convention of the literature in terms of the spaces used, in that the integration exponent in time and in $\omega$ may differ (in fact the latter will mostly be 2), hence the slightly different notation. Set $U_{p, \theta,(q)}^{\gamma}=L_{q}\left(\Omega, \mathcal{F}_{0}, \Psi^{1-2 / p} H_{p, \theta}^{\gamma-2 / p}\right)$ and let $\mathbb{H}_{p, \theta,(q)}^{\gamma}$ be the space of $\mathcal{P} \otimes \mathcal{B}(G)$-measurable functions belonging to $L_{q}\left(\Omega, L_{p}\left([0, T], H_{p, \theta}^{\gamma}\right)\right)$. Let furthermore $\mathfrak{H}_{p, \theta,(q)}^{\gamma} \subset \Psi \mathbb{H}_{p, \theta,(q)}^{\gamma}$ consist of functions $u$ for which there exists a $\psi \in U_{p, \theta,(q)}^{\gamma}, f \in \Psi^{-1} \mathbb{H}_{p, \theta,(q)}^{\gamma-2}$, and $g \in \mathbb{H}_{p, \theta,(q)}^{\gamma-1}$, such that for all $\varphi \in \mathcal{C}_{0}^{\infty}(G)$ the identity

$$
\left(u_{t}(\cdot), \varphi\right)=(\psi, \varphi)+\int_{0}^{t}\left(f_{s}(\cdot), \varphi\right) d s+\int_{0}^{t}\left(g_{s}^{k}(\cdot), \varphi\right) d W_{s}^{k}
$$

holds almost surely for all $t \in[0, T]$. We use the norm

$$
\|u\|_{\mathfrak{H}_{p, \theta,(q)}^{\gamma}}=\left\|\Psi^{-1} u\right\|_{\mathbb{H}_{p, \theta,(q)}^{\gamma}}+\|\psi\|_{U_{p, \gamma,(q)}^{\gamma}}+\|\Psi f\|_{\mathbb{H}_{p, \theta,(q)}^{\gamma-2}}+\|g\|_{\mathbb{H}_{p, \theta,(q)}^{\gamma-1}} .
$$

Let us recall some useful properties of these spaces. First of all, $\Psi^{-\alpha}$ is an isomorphism from $H_{p, \theta}^{\gamma}$ to $H_{p, \theta+\alpha p}^{\gamma}$. The following property, while we did not find 
explicitly stated elsewhere, follows easily from the definition (3), interpolation, and duality:

$$
\begin{aligned}
H_{p, \theta}^{\gamma} \subset H_{p}^{\gamma} & \text { if } \theta \leq d-(\gamma \vee 0) p, \\
H_{p}^{\gamma} \subset H_{p, \theta}^{\gamma} & \text { if } \theta \geq d-(\gamma \wedge 0) p .
\end{aligned}
$$

Finally, invoke from [11], Theorem 4.7, that for any $r^{\prime} \geq r \geq 2, \kappa \in[0,1]$, $2 / r<\beta \leq 1, q \in[0, r], \theta \in \mathbb{R}$ and $\gamma \in \mathbb{R}$, one has the continuous embedding

$$
\mathfrak{H}_{p, \theta,(q)}^{\gamma} \subset \Psi^{1-\gamma+(d-\theta) / p} L_{q}\left(\Omega, \mathcal{C}^{\alpha / 2-1 / p}\left([0, T], \mathcal{C}^{\gamma-\beta-d / p}(G)\right)\right),
$$

provided

$$
2 / p<\alpha<\beta \leq 1, \quad \gamma-\beta-d / p \in(0,1) .
$$

The following is a particular case of the of the quite general $L_{p}$-theory for SPDEs on domains from [8], Theorem 2.9.

THEOREM 4.1. Let Assumption 2.3(a) hold and assume that $f$ and $g$ do not depend on $u$ or $\nabla u$. Suppose furthermore that for some $c \in(0,1]$, Assumption 2.2 hold with $\kappa=c K$ and fix $p \geq 2$ and $\theta \in \mathbb{R}$ that satisfy

$$
d-1+p\left[1-\frac{1}{p(1-c)+c}\right]<\theta<d-1+p .
$$

Let $q \in[0, p], \gamma \in[0,4]$, and assume $\psi \in U_{p, \theta,(q)}^{\gamma}, f^{0} \in \Psi^{-1} \mathbb{H}_{p, \theta,(q)}^{\gamma-2}$, and $g \in$ $\mathbb{H}_{p, \theta,(q)}^{\gamma-1}$. Then the solution $u$ of (1) belongs to $\mathfrak{H}_{p, \theta,(q)}^{\gamma}$

$$
\|u\|_{\mathfrak{H}_{p, \theta,(q)}^{\gamma}} \leq N\left(\|\psi\|_{U_{p, \theta,(q)}^{\gamma}}+\|\Psi f\|_{\mathbb{H}_{p, \theta,(q)}^{\gamma-2}}+\|g\|_{\mathbb{H}_{p, \theta,(q)}^{\gamma-1}}\right),
$$

where $N$ depends on $\kappa, K, d, T, G, \theta, p$ and $q$.

REMARK 4.2. Notice that (12) is always satisfied if $d-2+p \leq \theta<d-1+p$.

REMARK 4.3. Both (11) and Theorem 4.1 are actually only stated in the references for the $q=p$ case. However, one can easily deduce the $q<p$ case using Lenglart's inequality; see [25], Proposition IV.4.7.

THEOREM 4.4. Let Assumptions 2.2, 2.3(a), 2.4 and 2.5 hold and let $u$ be the solution of (1) obtained from Theorem 3.2. Then for any $p \in[2, \infty)$, $u \in \mathfrak{H}_{p, d-2+p,(2)}^{\bar{v}}$, and in particular $u$ is a continuous random field in $Q$.

Proof. By Theorem 3.2, and (10), one has, for any $p \in[2, \infty)$,

$$
\begin{aligned}
\nabla \cdot \hat{f}(u) & \in L_{2}\left(\Omega, L_{p}\left([0, T], H_{p}^{-1}\right)\right) \subset L_{2}\left(\Omega, L_{p}\left([0, T], H_{p, d+p}^{-1}\right)\right) \\
& \subset L_{2}\left(\Omega, L_{p}\left([0, T], \Psi^{-1} H_{p, d-2+p}^{-1}\right)\right) \\
& \subset \Psi^{-1} \mathbb{H}_{p, d-2+p,(2)}^{-2+\bar{v}} .
\end{aligned}
$$


By similar reasoning and Assumption 2.4(b),

$$
\begin{aligned}
\left(\bar{f}(u)-f^{0}\right) & \in L_{2}\left(\Omega, L_{p}\left([0, T], L_{p}\right)\right) \\
& \subset L_{2}\left(\Omega, L_{p}\left([0, T], H_{p, d}^{0}\right)\right) \subset \mathbb{H}_{p, d-2+p,(2)}^{-1+\bar{v}} .
\end{aligned}
$$

Invoking also Assumption 2.5, one can therefore conclude that $f(u, \nabla u) \in$ $\Psi^{-1} \mathbb{H}_{p, d-2+p,(2)}^{-2+\bar{v}}$. A similar (in fact, easier) argument shows that $g(u) \in$ $\mathbb{H}_{p, d-2+p,(2)}^{-1+\bar{v}}$. Also,

$$
\psi \in L_{2}\left(\Omega, H_{p}^{\bar{v}}\right) \subset U_{\bar{p}, d-2+\bar{p},(2)}^{\bar{v}}
$$

by Assumption 2.5 and (10). Viewing $f(u, \nabla u)$ and $g(u)$ as fixed free terms, we can apply Theorem 4.1, with $\bar{v}$ in place of $\gamma$, and 2 in place of $q$, to obtain $u \in$ $\mathfrak{H}_{p, d-2+p,(2)}^{\bar{v}}$ as claimed. The second claim in the theorem follows by simply using (11).

Now that the basic interior regularity is quantified, we can prove Corollary 2.9.

Proof of Corollary 2.9. Let $(t, x),(s, y) \in Q_{T_{0}}$ and denote $\mid(t, x)-$ $(s, y) \mid=\varepsilon, d(x, \partial G) \vee d(y, \partial G)=\delta$. From Theorem 4.4 and (11), we can deduce that for any $\bar{\alpha}<\bar{v} / 3$ one has

$$
\left|u_{t}(x)-u_{s}(y)\right| \leq \eta_{0} \delta^{-\bar{v}} \varepsilon^{\bar{\alpha}}
$$

with some random variable $\eta_{0}$. Theorem 2.6 , on the other hand, yields that

$$
\left|u_{t}(x)-u_{s}(y)\right| \leq \eta_{1} \delta^{\alpha}
$$

with some random variable $\eta_{1}$. If $\delta \geq \varepsilon$, then this already gives the desired Hölder estimate. Otherwise set $\lambda=\alpha /(\alpha+\bar{v}) \in(0,1)$, and note that combining the two above bounds give

$$
\left|u_{t}(x)-u_{s}(y)\right| \leq \eta_{0}^{\lambda} \eta_{1}^{1-\lambda} \varepsilon^{\lambda \bar{\alpha}}
$$

as required.

Introduce for $T_{0} \geq 0, \alpha \geq 0$, the spaces $L_{\infty, \alpha}\left(Q_{T_{0}}\right)$, of functions $u \in L_{\infty}\left(Q_{T_{0}}\right)$ such that

$$
\|u\|_{L_{\infty, \alpha}\left(Q_{T_{0}}\right)}:=\sup _{(t, x) \in Q_{T_{0}}}|u(t, x)| d(x, \partial G)^{-\alpha}<\infty .
$$

It is easy to check that under the complex interpolation $[\cdot, \cdot]_{\theta}$ (for its definition see, e.g., [26]) these spaces behave as expected.

Proposition 4.5. Let $\alpha \neq \alpha^{\prime}, \theta \in(0,1)$, and $T_{0} \geq 0$. Then

$$
L_{\infty,(1-\theta) \alpha+\theta \alpha^{\prime}}\left(Q_{T_{0}}\right)=\left[L_{\infty, \alpha}\left(Q_{T_{0}}\right), L_{\infty, \alpha^{\prime}}\left(Q_{T_{0}}\right)\right]_{\theta} .
$$


Proof. Denote by $l_{\infty}^{\alpha}\left(L_{\infty}\right)$ the set of sequences with elements from $L_{\infty}\left(Q_{T_{0}}\right)$ such that

$$
\left\|\left(f_{n}\right)_{n \geq 0}\right\|_{l_{\infty}^{\alpha}\left(L_{\infty}\right)}=\sup _{n \geq 0} 2^{\alpha n}\left\|f_{n}\right\|_{L_{\infty}\left(Q_{T_{0}}\right)<\infty}
$$

Then the linear operators $S: L_{\infty, \alpha}\left(Q_{T_{0}}\right) \rightarrow l_{\infty}^{\alpha}\left(L_{\infty}\right), R: l_{\infty}^{\alpha}\left(L_{\infty}\right) \rightarrow L_{\infty, \alpha}\left(Q_{T_{0}}\right)$

$$
(S u)_{n}(t, x):=\mathbf{1}_{d(x, \partial G) \in\left[2^{-n-1}, 2^{-n}\right] \cdot \operatorname{diam}(G)} u_{t}(x), \quad(R(f))_{t}(x):=\sum_{n \geq 0} f_{n}(t, x)
$$

are bounded and satisfy $R S=\mathrm{id}$. The interpolation properties of the spaces $l_{\infty}^{\alpha}\left(L_{\infty}\right)$ (see [26], Theorem 1.18.2) then imply the claim, by [26], Theorem 1.2.4.

The setting of the aforementioned simpler version of Theorem 2.6 is then as follows.

Assumption 4.6. The function $f$ does not depend on $u$ and $\nabla u, g=0$, and almost surely

$$
\mathcal{K}_{2}:=\|\psi\|_{H_{d+3}^{1}}+\left\|f^{0}\right\|_{L_{\infty}\left([0, T], H_{d+3}^{1}\right)}<\infty
$$

THEOREM 4.7. Let Assumptions 2.2 and 2.3 hold and suppose $d_{1}<\infty$. Then there exists an $\alpha=\alpha\left(\kappa, K, d, d_{1}\right)>0$ such that for any $T_{0}>0$ there exists an almost surely finite random variable $\eta_{T_{0}}$ such that for all $\psi, f, g$ satisfying Assumption 4.6, the unique solution $u$ of (1) belongs to $L_{\infty, \alpha}\left(Q_{T_{0}}\right)$, and one has the bound

$$
\|u\|_{L_{\infty, \alpha}}\left(Q_{T_{0}}\right) \leq \eta_{T_{0}} \mathcal{K}_{2}
$$

Moreover, for fixed $K, d, d_{1}$, there exists a $c_{0}$ such that for sufficiently small $\kappa$ one has $\alpha>e^{-c_{0} / \kappa}$.

\section{LEMMA 4.8. Theorem 4.7 implies Theorem 2.6.}

PROOF. We only detail that the existence of the positive decay exponent $\alpha$ in Theorem 4.7 implies the corresponding statement in Theorem 2.6, the analogous implication concerning the exponential lower bound follows very similarly.

Fix $T_{0}>0$ and set, for $c \in[0, \infty], \Omega_{c}:=\left\{\eta_{T_{0}} \leq c\right\}$. Let, for $C \geq 1$ and $c \in$ $[0, \infty]$, denote by $\mathcal{S}_{c}^{C}(\bar{\psi}, \bar{f}, \bar{g})$ the random field $v \mathbf{1}_{\Omega_{c}}$, where $v$ solves

$$
\begin{aligned}
d v & =\left(C a^{i j} D_{i} D_{j} v+\bar{f}\right) d t+\left(\sigma^{i k} D_{i} v+\bar{g}^{k}\right) d W_{t}^{k} \quad \text { on } \mathbb{R}_{+} \times G, \\
v & =0 \quad \text { on } \mathbb{R}_{+} \times \partial G, \\
v_{0} & =\bar{\psi} \quad \text { on } G .
\end{aligned}
$$

When $C=1$ and/or $c=\infty$, the corresponding index will be dropped. 
Theorem 4.7 implies that for any $c<\infty, \mathcal{S}_{c}(\bar{\psi}, \bar{f}, 0)$ is bounded as an operator from $L_{\infty}\left(\Omega, H_{d+3}^{1}\right) \times L_{\infty}\left(\Omega, L_{\infty}\left([0, T], H_{d+3}^{1}\right)\right) \quad$ to $\quad L_{\infty}\left(\Omega, L_{\infty, \alpha}\left(Q_{T_{0}}\right)\right)$. Theorem 3.2 implies that $\mathcal{S}(\bar{\psi}, \bar{f}, 0)$ [and hence obviously also $\mathcal{S}_{c}(\bar{\psi}, \bar{f}, 0)$ for any $c<\infty]$ is bounded

$$
\text { from } L_{p}\left(\Omega, L_{\infty}(G)\right) \times L_{p}\left(\Omega, L_{d+3}\left([0, T], H_{d+3}^{-1}\right)\right) \quad \text { to } \quad L_{p}\left(\Omega, L_{\infty, 0}\left(Q_{T_{0}}\right)\right) \text {, }
$$

for any $p \in(0, \infty)$. Hence, by interpolation, $\mathcal{S}_{c}(\bar{\psi}, \bar{f}, 0)$ is also bounded

$$
\text { from } L_{2}\left(\Omega, H_{d+4}^{\gamma}\right) \times L_{2}\left(\Omega, L_{d+4}\left([0, T], H_{d+4}^{-1+\gamma}\right)\right) \quad \text { to } \quad L_{2}\left(\Omega, L_{\infty, \alpha^{\prime}}\left(Q_{T_{0}}\right)\right) \text {, }
$$

where $\gamma \leq \bar{v} \wedge 1 /(4(d+4)), \alpha^{\prime}>0$ depends only on $\alpha, \bar{v}$ and $d$. Note now that one has the identity

(13) $\mathcal{S}_{c}(\bar{\psi}, \bar{f}, \bar{g})=\mathcal{S}_{c}\left(\bar{\psi}, \bar{f}+(C-1) a^{i j} D_{i} D_{j}\left(\mathcal{S}^{C}(0,0, \bar{g})\right), 0\right)+\mathcal{S}_{c}^{C}(0,0, \bar{g})$.

By Theorem 4.1, for sufficiently large $C=C(d), S^{C}(0,0, \bar{g})$ is bounded

Notice that

$$
\text { from } \mathbb{H}_{d+4, d-1 / 2,(2)}^{\gamma} \quad \text { to } \quad \mathfrak{H}_{d+4, d-1 / 2,(2)}^{1+\gamma} \text {. }
$$

$$
\begin{aligned}
\mathfrak{H}_{d+4, d-1 / 2,(2)}^{1+\gamma} & \subset \Psi \mathbb{H}_{d+4, d-1 / 2,(2)}^{1+\gamma}=\mathbb{H}_{d+4,-4-1 / 2,(2)}^{1+\gamma} \\
& \subset L_{2}\left(\Omega, L_{d+4}\left([0, T], H_{d+4}^{1+\gamma}\right)\right),
\end{aligned}
$$

where for the last inclusion we used (10) and the condition on $\gamma$. It is known (see [22], Theorem 3.1) that $a^{i j} D_{i} D_{j}$ maps $H_{d+4}^{1+\gamma}$ to $H_{d+4}^{-1+\gamma}$. Therefore, the first term in (13) is bounded

$$
\begin{aligned}
& \text { from } L_{2}\left(\Omega, H_{d+4}^{\gamma}\right) \times L_{2}\left(\Omega, L_{d+4}\left([0, T], H_{d+4}^{-1+\gamma}\right)\right) \times \mathbb{H}_{d+4, d-1 / 2,(2)}^{\gamma} \\
& \quad \text { to } L_{2}\left(\Omega, L_{\infty, \alpha^{\prime}}\left(Q_{T_{0}}\right)\right)
\end{aligned}
$$

Finally, (11) implies that for a sufficiently small $\varepsilon=\varepsilon(d, \gamma)>0, \mathfrak{H}_{d+4, d-1 / 2,(2)}^{1+\gamma}$ is embedded into $\Psi^{\varepsilon} L_{2}\left(\Omega, L_{\infty}(Q)\right)$, and thus (possibly after lowering the value of $\left.\alpha^{\prime}\right)$ the whole solution map $\mathcal{S}_{c}(\bar{\psi}, \bar{f}, \bar{g})$ has boundedness in property in (14).

Since on $\Omega_{c}, u=\mathcal{S}(\psi, f(u, \nabla u), g(u))$, and by assumption $\psi \in L_{2}\left(\Omega, H_{d+4}^{\gamma}\right)$, it suffices to check that

$$
f(u, \nabla u) \in L_{2}\left(\Omega, L_{d+4}\left([0, T], H_{d+4}^{-1+\gamma}(G)\right)\right), \quad g(u) \in \mathbb{H}_{d+4, d-1 / 2,(2)}^{\gamma} .
$$

The first of these inclusions already follows from Theorem 3.2: by assumption, $f^{0} \in L_{2}\left(\Omega, L_{d+4}\left([0, T], H_{d+4}^{-1+\gamma}(G)\right)\right)$, we have already seen that $\left|\bar{f}(u)-f^{0}\right| \leq$ $K|u|^{m} \in L_{2}\left(\Omega, L_{\infty}(Q)\right)$, and note that

$$
\nabla \cdot \hat{f}(u) \in L_{2}\left(\Omega, L_{2}(Q)\right) \cap L_{2}\left(\Omega, L_{\infty}\left([0, T], H_{\infty}^{-1}(G)\right)\right)
$$

implies, by interpolation, $\nabla \cdot \hat{f}(u) \in L_{2}\left(\Omega, L_{d+4}\left([0, T], H_{d+4}^{-1+\gamma}(G)\right)\right)$. The second inclusion on (15) is a consequence of the Lipschitz continuity in $u$ of $g(u)$, the assumption $g^{0} \in \mathbb{H}_{d+4, d-1 / 2,(2)}^{\gamma}$, and that by Theorem 4.4,

$$
u \in \mathfrak{H}_{d+4, d-2+d+4,(2)}^{\gamma} \subset \mathbb{H}_{d+4, d-2,(2)}^{\gamma} .
$$


4.2. An Itô-Wentzell formula. In light of Lemma 4.8, we consider

$$
\begin{aligned}
d u_{t}(x)= & \left(a_{t}^{i j}(x) D_{i} D_{j} u_{t}(x)+f_{t}(x)\right) d t \\
& +\sigma_{t}^{i k}(x) D_{i} u_{t}(x) d W_{t}^{k} \quad \text { on } \mathbb{R}_{+} \times G, \\
u_{t}(x)= & 0 \quad \text { on } \mathbb{R}_{+} \times \partial G, \\
u_{0}(x)= & \psi(x) \quad \text { on } G .
\end{aligned}
$$

Consider the flow $\left(X_{t}(x)\right)_{(t, x) \in Q^{+}}$given by the SDE

$$
d X_{t}=-\sigma_{t}^{k}\left(X_{t}\right) d W_{t}^{k}
$$

which exists under Assumption 2.3(a) by the general theory of stochastic flows; see, for example, [19], Theorem II.3.1. Here and below $\sigma^{k}$ stands for the column vector $\left(\sigma^{1 k}, \ldots, \sigma^{d k}\right)$. Since the coefficients are assumed to vanish outside $G^{+}$, the flow $X$, and in fact any flow appearing below that is built from the coefficients $a$ and $\sigma$, are trivial outside $G^{+}$. Formally applying the Itô-Wentzell formula, the field $v_{t}(x):=u_{t}\left(X_{t}(x)\right)$ is expected to satisfy

$$
\begin{aligned}
\partial_{t} v_{t}(x)= & \bar{a}_{t}^{i j}\left(X_{t}(x)\right)\left(D_{i} D_{j} u_{t}\right)\left(X_{t}(x)\right)-\left(\sigma_{t}^{i k} D_{i} \sigma_{t}^{j k}\right)\left(X_{t}(x)\right)\left(D_{j} u_{t}\right)\left(X_{t}(x)\right) \\
& +f_{t}\left(X_{t}(x)\right) \\
= & \alpha_{t}^{i j}(x) D_{i} D_{j} v_{t}(x)+\beta_{t}^{i}(x) D_{i} v_{t}(x)+\varphi_{t}(x)
\end{aligned}
$$

on the (random) domain

$$
\tilde{Q}:=\left\{(t, x): t \in(0, T], X_{t}(x) \in G\right\},
$$

where here and in the following we use the notation

$$
\begin{aligned}
& \alpha_{t}(x)=\alpha_{t}(x)(\omega)=\left(\nabla X_{t}(x)\right)^{-1} \bar{a}_{t}\left(X_{t}(x)\right)\left(\left(\nabla X_{t}(x)\right)^{*}\right)^{-1}, \\
& \beta_{t}(x)=\beta_{t}(x)(\omega)=\left(\nabla X_{t}(x)\right)^{-1}\left(\Sigma_{t}\left(X_{t}(x)\right)-\nabla^{2}\left(X_{t}(x)\right) \alpha_{t}(x)\right), \\
& \varphi_{t}(x)=\varphi_{t}(x)(\omega)=f_{t}\left(X_{t}(x)\right) \\
& \Sigma_{t}(x)=\left(\nabla \sigma_{t}(x)\right) \sigma_{t}^{*}(x) .
\end{aligned}
$$

Unfortunately, no version of the Itô-Wentzell formula known to the author is actually applicable here, so we should confirm that the above formal computation is correct. It is worth noting that (again due to [19], Theorem II.3.1) the coefficients $\alpha$ and $\beta$ are both almost surely uniformly (in $t$ ) bounded in $\mathcal{C}^{2+\nu / 2}$ and $\mathcal{C}^{1+\nu / 2}$, respectively.

LEMMA 4.9. Let Assumptions 2.2, 2.3(a) and 4.6 hold. Then with the above notation, for almost all $\omega \in \Omega$, the function $\left(v_{t}(x)\right)_{(t, x) \in \tilde{Q}(\omega)}(\omega)$ is the probabilistic solution of (17) on $\tilde{Q}(\omega)$, with initial condition $\psi$ and boundary condition 0 . 
PROOF. Recall a Feynman-Kac formula for SPDEs with Dirichlet boundary conditions from [5]. Let $\left(B_{t}^{r}\right)_{r=1, \ldots, d, t \geq 0}$ be the canonical $d$-dimensional Wiener process on the standard Wiener space $\left(\hat{\Omega},\left(\hat{\mathcal{F}}_{t}\right)_{t \geq 0}, \hat{P}\right)$. Fix for now and for the rest of the paper $\rho$ to be a $\mathcal{C}^{2+v}(G)$ square root of $2 \bar{a}$. Introducing the flow $Y$ given by the SDE given on the completion of the probability space $(\Omega \times \hat{\Omega}$, $\left.\left(\mathcal{F}_{t} \otimes \hat{\mathcal{F}}_{t}\right)_{t \geq 0}, P \otimes \hat{P}\right)$,

$$
d Y_{t}=\left(\sigma_{t}^{i k} D_{i} \sigma_{t}^{k}+\rho_{t}^{i r} D_{i} \rho_{t}^{r}\right)\left(Y_{t}\right) d t-\sigma_{t}^{k}\left(Y_{t}\right) d W_{t}^{k}-\rho_{t}^{r}\left(Y_{t}\right) d B_{t}^{r}
$$

and the exit time of the inverse characteristics

$$
\gamma_{t, x}=\sup \left\{s \in[0, t]:\left(s, Y_{s, t}^{-1}(x)\right) \notin(0, T] \times G\right\},
$$

one has by [5], Theorem 2.1, for all $t \in[0, T], d x \otimes d P$-almost everywhere

$$
u_{t}(x)=\mathbb{E}^{\hat{P}}\left(\psi\left(Y_{0, t}^{-1}(x)\right) \mathbf{1}_{\gamma_{t, x}=0}+\int_{\gamma_{t, x}}^{t} f_{s}\left(Y_{s, t}^{-1}(x)\right) d s\right) .
$$

For a fixed $s \in[0, T]$, consider $w=\left(w^{(1)}, \ldots, w^{(d)}\right)$, the solution of the (system of) fully degenerate SPDEs

$$
\begin{aligned}
d w_{t}^{(l)}(x)= & a_{t}^{i j}(x) D_{i} D_{j} w_{t}^{(l)}(x) d t+\sigma_{t}^{i k}(x) D_{i} w_{t}^{(l)}(x) d W_{t}^{k} \\
& +\rho_{t}^{i r}(x) D_{i} w_{t}^{(l)}(x) d B_{t}^{r}
\end{aligned}
$$

on $[s, T] \times \mathbb{R}^{d}$, with initial condition $w_{s}^{(l)}(x)=x^{l}, l=1, \ldots, d$, which exists and is unique by [6]. Now we may apply the Itô-Wentzell formula [15], Theorem 1.1, and verify that the differential of $w_{t}^{(l)}\left(Y_{s, t}(x)\right)$ is 0 , and hence $w_{t}^{(l)}(x):=\left(Y_{s, t}^{-1}(x)\right)^{l}$. Applying the Itô-Wentzell formula again, one sees that $z_{t}^{(l)}(x):=w_{t}^{(l)}\left(X_{t}(x)\right)$ satisfies, with the notation $\bar{\rho}_{t}(x)=\left(\nabla X_{t}(x)\right)^{-1} \rho_{t}\left(X_{t}(x)\right)$,

$$
\begin{aligned}
d z_{t}^{(l)}(x)= & {\left[\bar{a}_{t}^{i j}\left(X_{t}(x)\right) D_{i} D_{j} w_{t}^{(l)}\left(X_{t}(x)\right)\right.} \\
& \left.-\left(\sigma_{t}^{i k} D_{i} \sigma_{t}^{j k}\right)\left(X_{t}(x)\right) D_{j} w_{t}^{(l)}\left(X_{t}(x)\right)\right] d t \\
& +\rho_{t}^{i r}\left(X_{t}(x)\right) D_{i} w_{t}^{(l)}\left(X_{t}(x)\right) d B_{t}^{r} \\
= & {\left[\alpha_{t}^{i j}(x) D_{i} D_{j} z_{t}^{(l)}(x)+\beta_{t}^{i}(x) D_{i} z_{t}^{(l)}(x)\right] d t+\bar{\rho}_{t}^{i r}(x) D_{i} z_{t}^{(l)}(x) d B_{t}^{r} }
\end{aligned}
$$

on $[s, T] \times \mathbb{R}^{d}$ with initial condition $z_{s}^{(l)}(x)=X_{s}^{l}(x)$. Note that (due to again [19], Theorem II.3.1) the coefficients $\alpha, \beta, \bar{\rho}$ are almost surely bounded processes in $\mathcal{C}^{2+v / 2}, \mathcal{C}^{1+v / 2}$, and $\mathcal{C}^{2+v / 2}$, respectively. So (see, e.g., [12]) one can find processes $\beta^{[m]}$ and $\bar{\rho}^{[m]}$ which are step functions in the sense that they are of the form

$$
\sum_{i=1}^{k} \sum_{j=1}^{l_{i}} \mathbf{1}_{\left[t_{i-1}, t_{i}\right)} \mathbf{1}_{A_{j}^{i}} h_{i, j}
$$


with some $k=k(m), l_{i}=l_{i}(m)$, some partition $0=t_{0}<t_{1}<\cdots<t_{k}=T$, some $\mathcal{F}_{t_{i-1}}$-measurable events $A_{j}^{i}$, and some deterministic smooth functions $h_{i, j}$, and such that furthermore

$$
\left\|\left(\beta^{[m]}-\beta\right)\right\|_{\mathcal{C}^{1+v / 3}}+\left\|\left(\bar{\rho}^{[m]}-\bar{\rho}\right)\right\|_{\mathcal{C}^{2+v / 3}} \rightarrow 0
$$

as $m \rightarrow 0$ in measure with respect to $d t \otimes d P$. One can of course also assume that the left-hand side above never exceeds 1 . Denote by $z^{[m]}$ the solution of (18) with $\alpha=\bar{\rho} \bar{\rho}^{*}, \beta$ and $\bar{\rho}$ replaced by $\bar{\rho}^{[m]}\left(\bar{\rho}^{[m]}\right)^{*}, \beta^{[m]}$ and $\bar{\rho}^{[m]}$, respectively. If we set $\tau_{n}:=\inf \left\{t \geq 0:\left|\left(\nabla X_{t}(x)\right)^{-1}\right|+\left|D_{k} X_{t}(x)\right| \leq n, \forall|k| \leq 3\right\} \wedge T$, then up to $\tau_{n}$, the coefficients are bounded in the appropriate spaces, and the existence an uniqueness of such solution on $\left[0, \tau_{n}\right]$ follows again from [6], along with the fact that

$$
\left\|z^{[m]}-z\right\|_{L_{q}\left(\llbracket 0, \tau_{n} \rrbracket, H_{p}^{1}\right)} \rightarrow 0
$$

as $m \rightarrow \infty$, for any $p, q \in[2, \infty)$.

Now introduce the flow $Z^{[m]}$ given by the SDE on the probability space $(\Omega \times$ $\left.\hat{\Omega},\left(\mathcal{F}_{t} \otimes \hat{\mathcal{F}}_{t}\right)_{t \geq 0}, P \otimes \hat{P}\right)$

$$
d Z_{t}^{[m]}=\left(-\beta_{t}^{[m]}+\bar{\rho}_{t}^{[m], i r} D_{i} \bar{\rho}_{t}^{[m], r}\right)\left(Z_{t}^{[m]}\right) d t-\bar{\rho}_{t}^{[m], r}\left(Z_{t}^{[m]}\right) d B_{t}^{r} .
$$

For almost all fixed $\omega, Z^{[m]}(\omega)$, as a function of $\hat{\omega}, s, t, x$, is the flow given by the $\operatorname{SDE}(21)$ on the probability space $\left(\hat{\Omega},\left(\hat{\mathcal{F}}_{t}\right)_{t \geq 0}, \hat{P}\right)$, with "deterministic" coefficients $\beta^{[m]}(\omega), \bar{\rho}^{[m]}(\omega)$. Moreover, the convergence (19) (at least along a subsequence) holds for almost all $\omega$ in measure with respect to $d t$. So by the limit theorems of flows (see, e.g., [20]), the $\operatorname{limit} Z:=\lim Z^{[m]}$ exists (e.g., in $\mathcal{C}^{v / 4}\left(Q^{+}\right)$), and is on the one hand the flow corresponding to the equation

$$
d Z_{t}=\left(-\beta_{t}+\bar{\rho}_{t}^{i r} D_{i} \bar{\rho}_{t}^{r}\right)\left(Z_{t}\right) d t-\bar{\rho}_{t}^{r}\left(Z_{t}\right) d B_{t}^{r}
$$

on $\left(\Omega \times \hat{\Omega},\left(\mathcal{F}_{t} \otimes \hat{\mathcal{F}}_{t}\right)_{t \geq 0}, P \otimes \hat{P}\right)$, and on the other hand, also on $\left(\hat{\Omega},\left(\hat{\mathcal{F}}_{t}\right)_{t \geq 0}, \hat{P}\right)$, for almost all $\omega \in \Omega$. One more application of the Itô-Wentzell formula then yields that the differential of $z_{t}^{[m]}\left(Z_{s, t}^{[m]}(x)\right)$ is 0 , that is, $z_{t}^{[m]}\left(Z_{s, t}^{[m]}(x)\right)=X_{s}(x)$. After passing to the limit using (20), and using the fact that both sides are continuous in all arguments, we therefore obtain that almost surely for all $0 \leq s \leq t \leq T, x \in \mathbb{R}^{d}$,

$$
Y_{s, t}^{-1}\left(X_{t}\left(Z_{s, t}(x)\right)\right)=X_{s}(x), \quad \text { or, } \quad Y_{s, t}^{-1}\left(X_{t}(x)\right)=X_{s}\left(Z_{s, t}^{-1}(x)\right) .
$$

By [19], Theorem II.6.1, for each fixed $\omega$ the inverse flow of $Z(\omega)$ can be given explicitly: $Z_{s, t}^{-1}(\omega)=U_{t, s}(\omega)$, where the flow $U=U(\omega)$ goes backwards in time and is given by the SDE (parametrized by $\omega \in \Omega$ )

$$
d U_{t}=\beta_{t}\left(U_{t}\right) d t+\bar{\rho}_{t}^{r}\left(U_{t}\right) d \overleftarrow{B}_{t}^{r}
$$

Furthermore, almost surely

$$
\begin{aligned}
\tau_{t, x} & :=\gamma_{t, X_{t}(x)}=\sup \left\{s \in[0, t]:\left(s, X_{S}\left(Z_{s, t}^{-1}(x)\right)\right) \notin(0, T] \times G\right\} \\
& =\sup \left\{s \in[0, t]:\left(s, Z_{s, t}^{-1}(x)\right) \notin \tilde{Q}\right\} \\
& =\sup \left\{s \in[0, t]:\left(s, U_{t, s}(x)\right) \notin \tilde{Q}\right\}
\end{aligned}
$$


is indeed the exit time of $U$ from $\tilde{Q}$. Hence

$$
\begin{aligned}
v_{t}(x) & =u_{t}\left(X_{t}(x)\right) \\
& =\mathbb{E}^{\hat{P}}\left(\psi\left(Y_{t}^{-1}\left(X_{t}(x)\right)\right) \mathbf{1}_{\gamma_{t, X_{t}(x)}=0}+\int_{\gamma_{t, X_{t}(x)}}^{t} f_{s}\left(Y_{s, t}^{-1}\left(X_{t}(x)\right)\right) d s\right) \\
& =\mathbb{E}^{\hat{P}}\left(\psi\left(U_{t, 0}(x)\right) \mathbf{1}_{\tau_{t, x}=0}+\int_{\tau_{t, x}}^{t} \varphi_{s}\left(U_{t, s}\right) d s\right),
\end{aligned}
$$

and the right-hand side is indeed the probabilistic solution of (17) with initial condition $\psi$ and boundary condition 0 . While the above equality is a priori only justified for all $t \in[0, T], d x \otimes d P$-almost everywhere, since both sides are continuous in $(t, x) \in \tilde{Q}$, the equality holds $P$-almost surely for all $(t, x) \in \tilde{Q}$.

4.3. Krylov's square root law for inverse flows. Define, for $\left(x_{t}\right)_{t \in[0, T]} \in$ $\mathcal{C}([0, T], V)$, where $V$ is some normed vector space, for $c \in(0, \infty)$, and $t \in[0, T]$ the quantity

$$
N_{n}(x ., c, t)=\#\left\{k=0, \ldots, n: \underset{\left[t-2^{-k}, t\right]}{\operatorname{osc}} x .>c 2^{-k / 2}\right\},
$$

where the convention $x_{t}=x_{0}$ for $t \in[-1,0)$ is used. We will need a generalization of the following square root law.

THEOREM 4.10 ([14]). Let $\left(w_{t}\right)_{t \geq 0}$ be a 1-dimensional Wiener process. Then for all c, $T \in(0, \infty)$, almost surely

$$
\limsup _{n \rightarrow \infty} \sup _{t \in[0, T]} \frac{1}{n+1} N_{n}(w ., c, t)=\pi(c)
$$

with a deterministic function $\pi(c)$ that converges to 0 as $c \rightarrow \infty$.

First we prove the following auxiliary lemma. For deterministic $\sigma$, similar estimates often appear in rough path theory, but we could not find a version that implies this form. We therefore provide a proof (using in fact less regularity requirement on $\sigma$ than in, e.g., [4], Proposition 8.3).

LEMMA 4.11. Let $\lambda \in(0,1 / 2)$. Let $\sigma$ be a bounded predictable process with values in $\mathcal{C}^{1}\left(\mathbb{R}^{d}\right)$ that vanishes outside $G^{+}$and such that $\|\sigma\|_{\mathcal{C}^{\lambda}\left([0, T], L_{\infty}\left(\mathbb{R}^{d}\right)\right)}$ has finite moments of any order. Then with the flow $X$ given by (16), any $\varepsilon>0$ and $p \geq 0$,

$$
\mathbb{E}\left(\sup _{s, t \in[0, T] ; y \in G^{+}} \frac{\left|X_{t}(y)-X_{s}(y)+\sigma_{s}^{k}\left(X_{s}(y)\right)\left(W_{t}^{k}-W_{s}^{k}\right)\right|}{|t-s|^{(1+2 \lambda) / 2-2 \varepsilon}}\right)^{p} \leq N
$$

for a constant $N$ depending on $p, \lambda, \varepsilon, d, T, G$ and the bounds on $\sigma$. 
ProOF. We apply Lemma A.15 with $V=\mathcal{C}\left(G^{+}, \mathbb{R}^{d}\right)$,

$$
\begin{aligned}
D_{s, t} & =X_{t}(\cdot)-X_{s}(\cdot)+\sigma_{s}^{k}\left(X_{s}(\cdot)\right)\left(W_{t}^{k}-W_{s}^{k}\right), \\
E_{s, s^{\prime}, t, t^{\prime}} & =\left(\sigma_{s}^{k}\left(X_{s}(\cdot)\right)-\sigma_{s^{\prime}}^{k}\left(X_{s^{\prime}}(\cdot)\right)\right)\left(W_{t}^{k}-W_{t^{\prime}}^{k}\right) .
\end{aligned}
$$

Condition (37) is clearly satisfied. As for the bounds (38), first, using also the usual version of the Kolmogorov continuity theorem, we can write, for any $p \geq 1$ (up to constants depending on $p, d$ and $G$ )

$$
\begin{aligned}
\mathbb{E}\left|D_{s, t}\right|^{p}= & \mathbb{E}\left|\sup _{y \in G^{+}} \int_{s}^{t} \sigma_{r}^{k}\left(X_{r}(y)\right)-\sigma_{s}^{k}\left(X_{s}(y)\right) d W_{r}^{k}\right|^{p} \\
\leq & \mathbb{E}\left|\int_{s}^{t} \sigma_{r}^{k}\left(X_{r}\left(y_{0}\right)\right)-\sigma_{s}^{k}\left(X_{s}\left(y_{0}\right)\right) d W_{r}^{k}\right|^{p} \\
& +\sup _{y, y^{\prime} \in G^{+}} \frac{\mathbb{E}\left|\int_{s}^{t} \sigma_{r}^{k}\left(X_{r}(y)\right)-\sigma_{r}^{k}\left(X_{r}\left(y^{\prime}\right)\right)-\sigma_{s}^{k}\left(X_{s}(y)\right)+\sigma_{s}^{k}\left(X_{s}\left(y^{\prime}\right)\right) d W_{r}^{k}\right|^{p}}{\left|y-y^{\prime}\right|^{d+1}} \\
\leq & \mathbb{E}\left|\int_{s}^{t}\right| \sigma_{r}\left(X_{r}\left(y_{0}\right)\right)-\left.\left.\sigma_{s}\left(X_{s}\left(y_{0}\right)\right)\right|^{2} d r\right|^{p / 2} \\
& +\sup _{y, y^{\prime} \in G^{+}} \frac{\mathbb{E}\left|\int_{s}^{t}\right| \sigma_{r}\left(X_{r}(y)\right)-\sigma_{r}\left(X_{r}\left(y^{\prime}\right)\right)-\sigma_{s}\left(X_{s}(y)\right)+\left.\left.\sigma_{s}\left(X_{s}\left(y^{\prime}\right)\right)\right|^{2} d r\right|^{p / 2}}{\left|y-y^{\prime}\right|^{d+1}},
\end{aligned}
$$

where $y_{0} \in G^{+}$is arbitrary. Fix $\varepsilon^{\prime} \in(0,1 / 2-\lambda)$ and denote

$$
\begin{aligned}
& K=\|\sigma\|_{L_{\infty}\left(\Omega \times[0, T], \mathcal{C}^{1}\left(\mathbb{R}^{d}\right)\right)}, \\
& \eta_{1}=\|\sigma\|_{\mathcal{C}^{\lambda}\left([0, T], \mathcal{C}^{1}\left(\mathbb{R}^{d}\right)\right)}, \quad \eta_{2}=\|X\|_{\mathcal{C}^{1 / 2-\varepsilon^{\prime}}\left([0, T], \mathcal{C}^{1-\varepsilon^{\prime}}\left(G^{+}\right)\right)} .
\end{aligned}
$$

The latter random variable has finite moments of any order due to [19], Theorem II.2.1. One has

$$
\begin{aligned}
\left|\sigma_{r}\left(X_{r}\left(y_{0}\right)\right)-\sigma_{s}\left(X_{s}\left(y_{0}\right)\right)\right| & \leq \eta_{1}|r-s|^{\lambda}+\left|\sigma_{s}\left(X_{r}\left(y_{0}\right)\right)-\sigma_{s}\left(X_{s}\left(y_{0}\right)\right)\right| \\
& \leq \eta_{1}|r-s|^{\lambda}+K\left|X_{r}\left(y_{0}\right)-X_{s}\left(y_{0}\right)\right| \\
& \leq \eta_{1}|r-s|^{\lambda}+K \eta_{2}|r-s|^{1 / 2-\varepsilon^{\prime}} \\
& \leq\left(\eta_{1}+K T \eta_{2}\right)|r-s|^{\lambda} .
\end{aligned}
$$

As for the other term, first, using the same bound as above, with $y_{0}$ replaced by $y$ and $y^{\prime}$,

$$
\left|\sigma_{r}\left(X_{r}(y)\right)-\sigma_{s}\left(X_{s}(y)\right)\right|+\left|\sigma_{s}\left(X_{s}\left(y^{\prime}\right)\right)-\sigma_{r}\left(X_{r}\left(y^{\prime}\right)\right)\right| \leq 2\left(\eta_{1}+K T \eta_{2}\right)|r-s|^{\lambda} .
$$

On the other hand, one also has

$$
\left|\sigma_{r}\left(X_{r}(y)\right)-\sigma_{r}\left(X_{r}\left(y^{\prime}\right)\right)\right|+\left|\sigma_{s}\left(X_{S}(y)\right)-\sigma_{s}\left(X_{s}\left(y^{\prime}\right)\right)\right| \leq 2 K \eta_{2}\left|y-y^{\prime}\right|^{1-\varepsilon^{\prime}} .
$$

Therefore, we also have

$$
\begin{aligned}
& \left|\sigma_{r}\left(X_{r}(y)\right)-\sigma_{r}\left(X_{r}\left(y^{\prime}\right)\right)-\sigma_{s}\left(X_{s}(y)\right)+\sigma_{s}\left(X_{s}\left(y^{\prime}\right)\right)\right| \\
& \quad \leq \eta_{3}|r-s|^{\lambda(1-\varepsilon)}\left|y-y^{\prime}\right|^{\left(1-\varepsilon^{\prime}\right) \varepsilon}
\end{aligned}
$$


with some random variable $\eta_{3}$ with finite moments of any order. Choosing $p$ large enough so that $p\left(1-\varepsilon^{\prime}\right) \varepsilon \geq d+1$, the second term on the right-hand side of (24) is bounded by a constant times

$$
|s-t|^{p(1 / 2+\lambda(1-\varepsilon))},
$$

and hence, combining this with (25), we get

$$
\mathbb{E}\left|D_{s, t}\right|^{p} \leq C|s-t|^{p(1 / 2+\lambda(1-\varepsilon))}
$$

uniformly in $s$ and $t$. Moving to the second bound in (38), we have

$$
\mathbb{E}\left|E_{s, s^{\prime}, t, t^{\prime}}\right|^{p} \leq\left|t-t^{\prime}\right|^{p / 2} \mathbb{E}^{1 / 2} \sup _{y \in G^{+}}\left|\sigma_{s}\left(X_{S}(y)\right)-\sigma_{s^{\prime}}\left(X_{s^{\prime}}(y)\right)\right|^{2 p} .
$$

The second component on the right-hand side can be estimated exactly as above: the only difference is that since one does not integrate in time, there is no factor $\left|s-s^{\prime}\right|^{p / 2}$ appearing. One hence has

$$
\mathbb{E}\left|E_{s, s^{\prime}, t, t^{\prime}}\right|^{p} \leq C\left|t-t^{\prime}\right|^{p / 2}\left|s-s^{\prime}\right|^{p \lambda(1-\varepsilon)},
$$

and so one can set $\gamma=(1+2 \lambda) / 2-\varepsilon$ in Lemma A.15: for large enough $p$, the conditions on the exponents are satisfied and we get the claim.

We can now prove the desired square root law.

Lemma 4.12. Let $\sigma$ satisfy Assumption 2.3 and assume $d_{1}<\infty$. Then, with the flow $X$ given by (16), for all $c \in(0, \infty)$, almost surely

$$
\limsup _{n \rightarrow \infty} \sup _{t \in[0, T]} \frac{1}{n+1} N_{n}\left(\left(\nabla X_{t}^{-1}\right)^{-1} X_{\cdot}^{-1}, c, t\right) \leq \hat{\pi}(c)
$$

with a deterministic function $\hat{\pi}(c)$, that depends only on $K$ and $d_{1}$, and that converges to 0 as $c \rightarrow \infty$, where for each $t,\left(\nabla X_{t}^{-1}\right)^{-1} X^{-1}$ is viewed as a process with values in $\mathcal{C}\left(G^{+}, \mathbb{R}^{d}\right)$.

PROOF. First note that

$$
\begin{aligned}
A_{s, t}:= & \sup _{y \in G^{+}}\left|X_{s}(y)-X_{t}(y)\right| \\
\leq & K\left|W_{t}-W_{s}\right| \\
& +|t-s|^{(1+v) / 2} \\
& \times \sup _{s^{\prime}, t^{\prime} \in[0, T] ; y \in G^{+}} \frac{\left|X_{t^{\prime}}(y)-X_{s^{\prime}}(y)+\sigma_{s^{\prime}}\left(X_{s^{\prime}}(y)\right)\left(W_{t^{\prime}}-W_{s^{\prime}}\right)\right|}{\left|t^{\prime}-s^{\prime}\right|^{(1+v) / 2}} .
\end{aligned}
$$


Denote the second term on the right-hand side by $B_{s, t}$, and let

$$
\begin{aligned}
& S_{t}^{i}(c)=\left\{n \in \mathbb{N}: \underset{\left[t-2^{-n}, t\right]}{\operatorname{osc}} W^{i}>c 2^{-n / 2}\right\}, \quad i=1, \ldots, d_{1}, \\
& S_{t}^{0}(c)=\left\{n \in \mathbb{N}: \sup _{s \in\left[t-2^{-n}, t\right]} B_{s, t}>c 2^{-n / 2}\right\} .
\end{aligned}
$$

Since, due to Lemma 4.11, $B_{s, t} \leq|t-s|^{(1+v) / 2} \eta$ for all $s, t \in[0, T]$ with a finite random variable $\eta$, the quantity $\sup _{t \in[0, T]} \# S_{t}^{0}(c)$ is also a.s. finite. By Theorem 4.10,

$$
\limsup _{n \rightarrow \infty} \sup _{t \in[0, T]} \frac{1}{n+1} \#\left(S_{t}^{i}(c) \cap[0, n]\right)=\pi(c) .
$$

Note that one has

$$
\begin{aligned}
X_{t}^{-1}(x)-X_{s}^{-1}(x) & =X_{t}^{-1}\left(X_{s}\left(X_{s}^{-1}(x)\right)\right)-X_{t}^{-1}\left(X_{t}\left(X_{s}^{-1}(x)\right)\right) \\
& =\nabla X_{t}^{-1}(x)\left(X_{s}\left(X_{s}^{-1}(x)\right)-X_{t}\left(X_{s}^{-1}(x)\right)\right)+C_{s, t},
\end{aligned}
$$

where one has the estimates

$$
\begin{gathered}
\left|\left(X_{s}\left(X_{s}^{-1}(x)\right)-X_{t}\left(X_{s}^{-1}(x)\right)\right)\right| \leq A_{s, t}, \\
\left|C_{s, t}\right| \leq \sup _{(t, x) \in Q^{+}}\left|\nabla^{2} X_{t}^{-1}(x)\right|\left|X_{s}\left(X_{s}^{-1}(x)\right)-X_{t}\left(X_{s}^{-1}(x)\right)\right|^{2} \\
\leq A_{s, t}^{2} \sup _{(t, x) \in Q^{+}}\left|\nabla^{2} X_{t}^{-1}(x)\right| .
\end{gathered}
$$

Hence, for all $t \in[0, T]$,

$$
\sup _{s \in\left[t-2^{-n}, t\right]} \sup _{x \in G^{+}}\left|\left(\nabla X_{t}^{-1}(x)\right)^{-1}\left(X_{t}^{-1}(x)-X_{s}^{-1}(x)\right)\right| \leq \sup _{s \in\left[t-2^{-n}, t\right]}\left(A_{s, t}+A_{s, t}^{2} \xi\right)
$$

with some finite random variable $\xi$. So whenever $n \notin \bigcup_{i=0}^{d_{1}} S_{t}^{i}(c)$ and $c 2^{-n / 2} \leq$ $1 / \xi$, the right-hand side above is bounded by $2\left(K d_{1}+1\right) c 2^{-n / 2}$ for all $s \in$ $\left[t-2^{-n}, t\right]$, and so setting $\hat{\pi}(c)=d_{1} \pi\left(c /\left(4 K d_{1}+4\right)\right)$ completes the proof of the lemma.

REMARK 4.13. The square root law above is the only instance in the proof where the assumption $d_{1}<\infty$ is used. As mentioned in Remark 2.7, a slight extension is available: for any sequence $\left(c_{i}\right)_{i \in \mathbb{N}}$, if $n \notin S_{t}:=\bigcup_{i=1}^{\infty} S_{t}^{i}\left(c_{i}\right)$, then one has

$$
\sup _{s \in\left[t-2^{-n}, t\right]}\left|A_{s, t}-B_{s, t}\right| \leq 2^{-n / 2} \sup _{x \in G^{+}} \sum_{i=1}^{\infty} \sigma_{t}^{i}\left(X_{t}(x)\right) c_{i} .
$$

If $\tilde{c}_{i} \rightarrow \infty$ sufficiently fast so that $\sum_{i=1}^{\infty} \pi\left(\tilde{c}_{i}\right)<\infty$, then the upper density of $S_{t}$ with the choice $c_{i}=C \tilde{c}_{i}$ can be made arbitrarily small by choosing $C$ to be 
sufficiently large. Hence if the decay of $\sigma^{i}$ is so fast that $\sum_{i=1}^{\infty} \sigma^{i} \tilde{c}_{i}<\infty$ uniformly over all choice of arguments, then we obtain the square root law as before. Note however that this decay condition is far stronger than requiring $\sigma$ to be in $l_{2}$, or even $l_{1}$.

Our final lemma, which will essentially allow us to utilize the above square root law, is the following estimate for hitting probabilities.

LEMMA 4.14. Let $p \geq 0$ be an integer, $\left(\bar{B}_{t}\right)_{t \geq 0}$ be a d-dimensional Wiener process on a filtered probability space $\left(\bar{\Omega},\left(\overline{\mathcal{F}}_{t}\right)_{t \geq 0}, \bar{P}\right)$, and let

$$
\xi_{s}=\int_{0}^{s} b_{s^{\prime}} d s^{\prime}+\int_{0}^{s} a_{s^{\prime}} d \bar{B}_{s^{\prime}}
$$

with $a_{t}$ and $b_{t}$ being bounded predictable processes with values in $\mathbb{R}^{d \times d}$ and $\mathbb{R}^{d}$, respectively. Fix $c \geq 1, r \geq 7 c$, denote $Q_{r}^{p}:=\left[0,2^{-p}\right] \times B_{2^{-p / 2} r}, f i x(t, x) \in Q_{r}^{p+1}$ and assume that on $\left\{(s, \omega):\left(t+s, x+\xi_{s}\right) \in Q_{r}^{p}\right\}$ the bounds $\left|b_{s}(\omega)\right| \leq C 2^{p / 2}$ and

$$
\delta I \leq a_{s}(\omega) a_{s}^{*}(\omega) \leq \Delta I
$$

hold, the latter in the sense of positive semidefinite matrices, with some $C, \delta, \Delta>0$. Let furthermore $n \in \mathbb{R}^{d}$ be a unit vector, and $A \subset Q_{r}^{p}$ be a closed set such that $\left\{(s, y):\langle y, n\rangle \geq c 2^{-p / 2}\right\} \cap Q_{r}^{p} \subset A$. Finally, set

$$
\tau_{t, x}=\inf \left\{s>0:\left(t+s, x+\xi_{s}\right) \in A \cup \partial Q_{r}^{p}\right\} .
$$

Then one has

$$
P\left(\left(t+\tau_{t, x}, x+\xi_{\tau_{t, x}}\right) \notin A\right) \leq \gamma(c, r, d, \delta, \Delta, C)<1
$$

for some function $\gamma$, depending only on the indicated parameters. Moreover, for fixed $c, r, d, \Delta$, there exists a $c_{0}$ such that for sufficiently small $\delta$ one has $1-\gamma>$ $e^{-c_{0} / \delta}$

PROOF. By rotational symmetry, we may assume that $n=(-1,0, \ldots, 0)$ and by Brownian rescaling we may assume $p=0$. It is also clear that if $A$ is replaced by $\tilde{A}_{c} \cap \bar{Q}_{r}^{0}$, where

$$
\tilde{A}_{c}=\left\{(s, y): y \in A_{c}\right\}:=\left\{(s, y): y_{1} \leq-c\right\},
$$

both in the definition of $\tau$ and on the left-hand side of (27), then the left-hand side of (27) increases, so it suffices to prove the statement for $A=\tilde{A}_{c} \cap \bar{Q}_{r}^{0}$. One can also trivially assume $x \notin \operatorname{int} A_{c}$, since otherwise the left-hand side of (27) is just 0 .

Let $\varphi, \psi$ be smooth functions on $\mathbb{R}$ such that

$$
\begin{aligned}
& \text { for }|a| \leq 5 / 7 r, \quad \varphi(a)=\frac{1}{c+\sqrt{r^{2}-a^{2}}}, \quad \psi(a)=c ; \\
& \text { for }|a| \geq 6 / 7 r, \quad \varphi(a)=1, \quad \psi(a)=c+1 ; \\
& \text { for }|a| \in[5 / 7 r, 6 / 7 r], \quad \varphi(a) \in\left[\frac{1}{c+\sqrt{r^{2}-a^{2}}}, 1\right], \quad \psi(a) \in[c, c+1] .
\end{aligned}
$$


Denote $\tilde{y}:=\left(y_{2}, \ldots, y_{d}\right)$, and introduce the function $f(y)=\varphi(|\tilde{y}|)\left(y_{1}+\psi(|\tilde{y}|)\right)$, the process

$$
\hat{\xi}_{s}:=f(x)+\int_{0}^{s} \hat{b}_{s^{\prime}} d s^{\prime}+\int_{0}^{s} \hat{a}_{s^{\prime}} d \bar{B}_{s^{\prime}}:=f\left(x+\xi_{s}\right),
$$

and the stopping time

$$
\hat{\tau}:=\inf \left\{s>0:\left(t+s, \hat{\xi}_{s}\right) \in[0,1] \times\{0,1\} \cup\{1\} \times[0,1]\right\} .
$$

By construction, on $B_{r} \backslash \operatorname{int} A_{c}, f$ is nonnegative, and on $\left\{y:|y|=r, y_{1} \geq-c\right\}$, one has $f(y) \geq 1$. Therefore,

$$
\left\{\left(t+\tau_{t, x}, x+\xi_{\tau_{t, x}}\right) \notin \tilde{A}_{c} \cap \bar{Q}_{r}^{0}\right\} \subset\left\{\left(t+\hat{\tau}, \hat{\xi}_{\hat{\tau}}\right) \notin[0,1] \times\{0\}\right\} .
$$

Note also that

$$
|\nabla f(y)| \geq\left|D_{1} f(y)\right| \geq \inf _{a \in \mathbb{R}} \varphi(a)=\frac{1}{c+r},
$$

and so $|\hat{a}|^{2}$ is bounded from below $\frac{\delta}{(r+c)^{2}}$. It is also clear from Itô's formula that there exists a $\hat{C}=\hat{C}(r, c, d)$ such that $\sup _{s \in[0,1]}\left|\hat{b}_{s}\right| \leq \hat{C}(C+\Delta)$. Next, we claim that there exists an $m=m(r, c)<1$ such that for $y \in B_{2^{-1 / 2}}, f(y) \leq m$. Indeed, we can write

$$
\begin{aligned}
\max _{y_{1}^{2}+|\tilde{y}|^{2} \leq r^{2} / 2} f(y) & =\max _{y_{1}^{2}+|\tilde{y}|^{2} \leq r^{2} / 2} \frac{y_{1}+c}{c+\sqrt{r^{2}-|\tilde{y}|^{2}}} \\
& =\max _{y_{1}^{2}+|\tilde{y}|^{2}=r^{2} / 2} \frac{y_{1}+c}{c+\sqrt{r^{2}-|\tilde{y}|^{2}}} \\
& =\max _{y_{1}^{2} \leq r^{2} / 2} \frac{y_{1}+c}{c+\sqrt{r^{2} / 2+y_{1}^{2}}}=: \max _{y_{1}^{2} \leq r^{2} / 2} g\left(y_{1}\right) .
\end{aligned}
$$

Trivially, $\lim _{y_{1} \rightarrow \pm \infty} g\left(y_{1}\right)= \pm 1$, so it suffices to show that $g^{\prime}>0$. Direct calculation shows

$$
g^{\prime}\left(y_{1}\right)=\frac{c \sqrt{r^{2} / 2+y_{1}^{2}}-c y_{1}+r^{2} / 2}{\sqrt{r^{2} / 2+y_{1}^{2}}\left(\sqrt{r^{2} / 2+y_{1}^{2}}+c\right)^{2}} .
$$

If the numerator were 0 for some $y_{1}$, that would imply

$$
c^{2}\left(r^{2} / 2+y_{1}^{2}\right)=c^{2} y_{1}^{2}-c r^{2} y_{1}+r^{4} / 4,
$$

which gives $y_{1}=\frac{r^{2} / 4-c^{2} / 2}{c}$, but since substituting this back to (29) gives a positive quantity, we get the claim.

Let us now set $t_{0}=(1-m) /(2 \hat{C}(C+\Delta))$, so that one has

$$
\sup _{s \in\left[0, t_{0}\right]}\left|\int_{0}^{s} \hat{b}_{s^{\prime}} d s^{\prime}\right| \leq(1-m) / 2 .
$$


Define

$$
\begin{aligned}
& \tilde{\xi}_{s}:=f(x)+\int_{0}^{s} \hat{a}_{s^{\prime}} d \bar{B}_{s^{\prime}}, \\
& \tilde{\tau}:=\inf \left\{s>0:\left(t+s, \tilde{\xi}_{s}\right) \in\left[0, t+t_{0}\right] \times\left\{-\frac{1-m}{2}, \frac{1+m}{2}\right\} \cup\left\{t+t_{0}\right\} \times[0,1]\right\}
\end{aligned}
$$

and notice that

$$
\left\{\left(t+\hat{\tau}, \hat{\xi}_{\hat{\tau}}\right) \notin[0,1] \times\{0\}\right\} \subset\left\{\left(t+\tilde{\tau}, \tilde{\xi}_{\tilde{\tau}}\right) \notin\left[0, t+t_{0}\right] \times\left\{-\frac{1-m}{2}\right\}\right\} .
$$

The latter event is now in the scope of [14], Lemma 3.7: the process whose hitting time we are considering is a 1-dimensional continuous martingale with quadratic variation uniformly bounded from below, and the starting point $(t, f(x))$ is strictly separated from the right boundary $[0,1] \times\left\{\frac{1+m}{2}\right\}$. From $(28),(30)$ and the application of [14], Lemma 3.7, one thus has

$$
1-P\left(\left(t+\tau_{t, x}, x+\xi_{\tau_{t, x}}\right) \notin A\right) \geq P\left(\sup _{t \in\left[0, \tilde{t}_{0}\right]} w_{t} \leq a \delta^{-1 / 2}, \inf _{t \in\left[0, \tilde{t}_{0}\right]} w_{t} \leq b \delta^{-1 / 2}\right),
$$

where $w$ is a 1 -dimensional Wiener process, and the numbers $\tilde{t}_{0}, a,-b>0$ depend on $r, c, d, \Delta, C$. The right-hand side is clearly positive and the lower bound $e^{-c_{0} / \delta}$ bound follows from standard properties of the Wiener process.

4.4. Proof of Theorem 4.7. Throughout the proof, we work with a fixed $\omega \in \Omega$. By linearity, we can assume that $\psi, f \geq 0$, and hence also $u, v \geq 0$. We will throughout the proof often use the shorthand $z=(t, x)$.

Define $v^{\varepsilon}$ as the probabilistic solution of (17) on

$$
\tilde{Q}^{\varepsilon}:=\left\{z: t \in[0, T],\left(\zeta_{\varepsilon} 3 * X .(x)\right)_{t} \in\left(G+B_{\varepsilon}\right)\right\},
$$

with initial condition $\psi$ and boundary condition 0 , where $\zeta \in \mathcal{C}_{0}^{\infty}\left(\mathbb{R}_{+}\right)$and $\zeta_{\varepsilon}(s)=\varepsilon^{-1} \zeta\left(\varepsilon^{-1} s\right)$. Simply by the uniform in $x 1 / 2$-Hölder-continuity in time of $X$, there exists an $\varepsilon_{0}=\varepsilon_{0}(\omega)$ such that for all $0<\varepsilon<\varepsilon_{0}$, one has $\tilde{Q} \subset \tilde{Q}^{\varepsilon}$ and, therefore, $v \leq v^{\varepsilon}$. Moreover, $v^{\varepsilon}$ agrees with the classical solution of (17) with the same initial-boundary conditions and, therefore, it is continuously differentiable in time and twice continuously differentiable in space on the closure of $\left\{z: t \in\left[T_{0} / 2, T\right], x \in \tilde{Q}_{t}^{\varepsilon}\right\}$, where $\tilde{Q}_{t}^{\varepsilon}=\left\{x:(t, x) \in \tilde{Q}^{\varepsilon}\right\}$.

Using Lemma 4.12 and the notation in Lemma 4.14, fix a $c_{0}$ such that $1 / 2>\hat{\pi}\left(c_{0}\right)=: 1-\hat{\pi}$ and a $r_{0} \geq 7\left(2^{1 / 4} c_{0}+1\right)$, and set $\gamma:=\gamma\left(2^{1 / 4} c_{0}+1\right.$, $\left.r_{0}, d, \kappa 2^{1 / 2}, K 2^{1 / 2}, 1\right) \vee(1 / 2)<1$. Since $M(z):=\nabla X_{t}(x)$ is uniformly continuous and separated away from zero, there exists a $\delta_{0}=\delta_{0}(\omega)>0$ such that whenever $\left|z-z^{\prime}\right| \leq \delta_{0}$

$$
2^{-1 / 4} I \leq M(z) M^{-1}\left(z^{\prime}\right) \leq 2^{1 / 4} I
$$


Take any $\bar{t} \in\left[T_{0}, T\right]$. Let $p_{1}, p_{2} \ldots$, be the nonnegative integers such that

$$
\sup _{x \in G^{+}} \sup _{s, t \in\left[\bar{t}-2^{\left.-p_{i}, \bar{t}\right]}\right.}\left|M\left(\bar{t}, X_{\bar{t}}^{-1}(x)\right)\left(X_{t}^{-1}(x)-X_{s}^{-1}(x)\right)\right| \leq c_{0} 2^{-p_{i} / 2},
$$

and introduce, for integers $j \geq-\log _{2}\left(1 \wedge\left(T_{0} / 2\right)\right)$,

$$
S(j):=\left\{z: t \in\left[\bar{t}-2^{-j}, \bar{t}\right], x \in \tilde{Q}_{t}, d\left(M(z) x, M(z) \partial \tilde{Q}_{\bar{t}}\right) \leq r_{0} 2^{-j / 2}\right\}
$$

and $\mathcal{M}^{\varepsilon}(j):=\sup _{S_{j}}\left|v^{\varepsilon}\right|$, where for brevity we suppress the $\bar{t}$-dependence of these objects. Of course, $\left(\mathcal{M}^{\varepsilon}(j)\right)$ is a decreasing sequence. Suppose now that there exists $\bar{t}$-independent indices $j_{0}=j_{0}(\omega)$ and $j_{1}=j_{1}(\varepsilon, \omega)$ such that $j_{1} \rightarrow \infty$ as $\varepsilon \rightarrow 0$ almost surely and that for all $j_{1} \geq p_{i} \geq j_{0}$

$$
\mathcal{M}^{\varepsilon}\left(p_{i+1}\right) \leq 2^{-p_{i}} \mathcal{K}_{2}+\gamma \mathcal{M}^{\varepsilon}\left(p_{i}\right)
$$

By iterating the above, we get

$$
\mathcal{M}^{\varepsilon}\left(p_{i+1}\right) \leq \gamma^{i} \mathcal{K}_{2}+\gamma \mathcal{M}^{\varepsilon}\left(p_{i}\right) \leq \gamma^{i-j_{0}}\left(i \mathcal{K}_{2}+\mathcal{M}^{\varepsilon}(0)\right) \leq C \bar{\gamma}^{i} \mathcal{K}_{2}(2+T)
$$

with $\bar{\gamma}=\gamma / 2+1 / 2$ and some $C=C(\gamma, \omega)$. Denote by $j_{2}=j_{2}(\omega)$ the index such that for all $j \geq j_{2}$,

$$
\#\left\{i: p_{i} \leq j\right\} \geq j \hat{\pi} / 2,
$$

which exists and does not depend on $\bar{t}$ by the definition of $\hat{\pi}$. We therefore obtain, for $j_{1} \geq j \geq j_{2}$,

$$
\mathcal{M}^{\varepsilon}(j) \leq \mathcal{M}^{\varepsilon}\left(p_{j \hat{\pi} / 2}\right) \leq C(2+T) \mathcal{K}_{2}\left(\bar{\gamma}^{\hat{\pi} / 2}\right)^{j} .
$$

Denote $\hat{C}=C(2+T), \hat{\gamma}=\bar{\gamma}^{\hat{\pi} / 2}$. Note that for any $x \in G$, with

$$
\bar{\mu}:=\sup _{z, z^{\prime} \in Q^{+}}|M(z)|\left|M^{-1}\left(z^{\prime}\right)\right|,
$$

one has the trivial bound

$$
\begin{aligned}
d\left(M\left(\bar{t}, X_{\bar{t}}^{-1}(x)\right) X_{\bar{t}}^{-1}(x), M\left(\bar{t}, X_{\bar{t}}^{-1}(x)\right) \partial \tilde{Q}_{\bar{t}}\right) & \leq d(x, \partial G) \bar{\mu} \\
& =r_{0} 2^{-\left[2 \log _{2}\left(\frac{r_{0}}{d(x, \partial G) \bar{\mu}}\right)\right] / 2} .
\end{aligned}
$$

If $2 \log _{2}\left(\frac{r_{0}}{d(x, \partial G) \bar{\mu}}\right)>j_{2}$, choose $\varepsilon \leq \varepsilon_{0}$ such that $j_{1}(\varepsilon) \geq 2 \log _{2}\left(\frac{r_{0}}{d(x, \partial G) \bar{\mu}}\right)$, so that we can write

$$
\begin{aligned}
u_{\bar{t}}(x) & =v_{\bar{t}}\left(X_{\bar{t}}^{-1}(x)\right) \leq v_{\bar{t}}^{\varepsilon}\left(X_{\bar{t}}^{-1}(x)\right) \leq \mathcal{M}^{\varepsilon}\left(\left\lfloor 2 \log _{2}\left(\frac{r_{0}}{d(x, \partial G) \bar{\mu}}\right)\right\rfloor\right) \\
& \leq \hat{C} \mathcal{K}_{2} \hat{\gamma}^{\left\lfloor 2 \log _{2}\left(\frac{r_{0}}{d(x, \partial G) \bar{\mu}}\right)\right\rfloor} \\
& \leq \hat{C} \mathcal{K}_{2} \hat{\gamma}^{2 \log _{2} \frac{r_{0}}{\bar{\mu}}-2} d(x, \partial G)^{-2 \log _{2} \hat{\gamma}} .
\end{aligned}
$$

Note that-as claimed in the theorem-the exponent $\alpha:=-2 \log _{2} \hat{\gamma}>0$ of the decay depends only on $\kappa, K, d, d_{1}$. Moreover, the exponential (in $1 / \kappa$ ) 
lower bound on $\alpha$ follows from the corresponding statement of Lemma 4.14. If $2 \log _{2}\left(\frac{r_{0}}{d(x, \partial G) \bar{\mu}}\right) \leq j_{2}$ we can use the trivial bound

$$
u_{\bar{t}}(x) \leq \sup _{\tilde{Q}} v \leq \mathcal{K}_{2}(T+1) \leq \mathcal{K}_{2}(T+1)\left(\frac{2^{j_{2} / 2} \bar{\mu}}{r_{0}}\right)^{\alpha} d(x, \partial G)^{\alpha} .
$$

Since $\bar{t}$ was arbitrary, this yields the claim, so it would suffice to prove (32). By virtually the same argument, it is also enough (and will be more convenient) to prove

$$
\mathcal{M}^{\varepsilon}\left(p_{i+2}\right) \leq 2^{-p_{i}} \mathcal{K}_{2}+\gamma \mathcal{M}^{\varepsilon}\left(p_{i-1}\right)
$$

Recall that for any bounded $\mathcal{C}^{1}$ domain there exists a function $\varepsilon_{G}(\alpha):(0,1) \rightarrow$ $(0, \infty)$ such that for any $\alpha \in(0,1)$ and $x \in \partial G$ one has $B_{\varepsilon_{G}(\alpha)}(x) \cap\{y$ : $\left.\left\langle\frac{y-x}{|y-x|}, n_{x}\right\rangle \geq \alpha\right\} \subset G^{c}$, where $n_{x}$ is the normal derivative of $\partial G$ at $x$. Let then $j_{0}$ be the smallest integer such that for all $j \geq j_{0}$ :

(a) $2 r_{0} 2^{-j / 2} \tilde{\mu} \leq 1 /\left(32 r_{0}\right)$, where $\tilde{\mu}=\sup _{z, z^{\prime} \in Q^{+}}\left|\nabla^{2} X_{t}(x)\right|\left|M^{-1}(z)\right|^{2}$,

(b) $2 \bar{\mu} r_{0} \overline{2}^{-j / 2} \leq \varepsilon_{G}\left(1 / 16 r_{0}\right)$,

(c) $2^{-j}+\sup _{|s-t| \leq 2^{-j+1}} \sup _{y \in \mathbb{R}^{d}}\left|X_{t}^{-1} X_{s} y-y\right|+\bar{\mu} r_{0} 2^{-j / 2} \leq \delta_{0}$,

(d) $\bar{\mu} \sup _{(t, x) \in \tilde{Q}^{+}}\left|\beta_{t}(x)\right| \leq 2^{-j / 2}$.

That is of course equivalent to saying that $j_{0}$ is the smallest integer that satisfies (a)-(d). Clearly, $j_{0}$ has no dependence on $\bar{t}$.

Fix now $i$ such that $p_{i} \geq j_{0}$ as well as $0<\varepsilon<\varepsilon_{0}$ and fix also $z_{0}=\left(t_{0}, x_{0}\right) \in$ $S\left(p_{i+2}\right)$. Let $z^{\prime}=\left(\bar{t}, x^{\prime}\right)$, where $x^{\prime} \in \partial \tilde{Q}_{\bar{t}}$ is a minimizer of the distance between $M\left(z_{0}\right) x_{0}$ and $M\left(z_{0}\right) \partial \tilde{Q}_{\bar{t}}$. Recall the definition of the flow $U$ from (23) and introduce, with $M_{0}:=M\left(z^{\prime}\right)$,

$$
\begin{aligned}
& Q_{0}:=\left\{z: t \in\left[t_{0}-2^{-p_{i}}, t_{0}\right], d\left(M_{0} x, M_{0} x^{\prime}\right) \leq r_{0} 2^{-p_{i} / 2}\right\}, \\
& A_{0}:=Q_{0} \backslash \tilde{Q}^{\varepsilon}, \quad \tau_{0}:=\sup \left\{s<t_{0}:\left(s, U_{t_{0}, s}\left(x_{0}\right)\right) \in A_{0} \cup \partial Q_{0}\right\} .
\end{aligned}
$$

One has $z_{0} \in Q_{0}$, in fact, even

$$
\left|M_{0} x_{0}-M_{0} x^{\prime}\right| \leq 2^{1 / 4}\left|M\left(z_{0}\right) x_{0}-M\left(z_{0}\right) x^{\prime}\right| \leq r_{0} 2^{-p_{i+2} / 2+1 / 4} \leq r_{0} 2^{-\left(p_{i+1}\right) / 2} .
$$

Since $v^{\varepsilon}$ is sufficiently smooth on the closure of $Q_{0} \cap \tilde{Q}^{\varepsilon}$, by Itô's formula one has

$$
v_{t_{0}}^{\varepsilon}\left(x_{0}\right)=\mathbb{E}^{\hat{P}}\left(\int_{\tau_{0}}^{t_{0}} \varphi_{s}\left(U_{t_{0}, s}\left(x_{0}\right)\right) d s+v_{\tau_{0}}^{\varepsilon}\left(U_{t_{0}, \tau_{0}}\left(x_{0}\right)\right)\right) .
$$

If $z_{\tau_{0}}:=\left(\tau_{0}, U_{t_{0}, \tau_{0}}\left(x_{0}\right)\right) \in A_{0}$, then $v^{\varepsilon}\left(z_{\tau_{0}}\right)=0$. If however $z_{\tau_{0}} \notin A_{0}$, then we claim that $z_{\tau_{0}} \in S\left(p_{i-1}\right)$. Indeed, first note that since one cannot exit $\tilde{Q}$ without 
exiting $\tilde{Q}_{\varepsilon}$ first, if $z_{\tau_{0}} \notin A_{0}$, then one has $U_{t_{0}, \tau_{0}}\left(x_{0}\right) \in \tilde{Q}_{\tau_{0}}$. Next, by property (c) of $j_{0}$, one has $\left|z_{\tau_{0}}-z^{\prime}\right| \leq \delta_{0}$, and hence

$$
\begin{aligned}
d\left(M\left(z_{\tau_{0}}\right) U_{t_{0}, \tau_{0}}\left(x_{0}\right), M\left(z_{\tau_{0}}\right) \partial \tilde{Q}_{\bar{t}}\right) & \leq 2^{1 / 4} d\left(M_{0} U_{t_{0}, \tau_{0}}\left(x_{0}\right), M_{0} \partial \tilde{Q}_{\bar{t}}\right) \\
& \leq 2^{1 / 4}\left|M_{0} U_{t_{0}, \tau_{0}}\left(x_{0}\right)-M_{0} x^{\prime}\right| \\
& \leq 2^{1 / 4} r_{0} 2^{-p_{i} / 2} \leq r_{0} 2^{-p_{i-1} / 2} .
\end{aligned}
$$

As for the time-coordinate, one simply has

$$
\tau_{0} \geq t_{0}-2^{-p_{i}} \geq \bar{t}-2^{-p_{i}}-2^{-p_{i}} \geq \bar{t}-2^{-p_{i-1}},
$$

as required. Hence,

$$
v_{t_{0}}^{\varepsilon}\left(x_{0}\right) \leq 2^{-p_{i}} \mathcal{K}_{2}+\hat{P}\left(\left(\tau_{0}, U_{t_{0}, \tau_{0}}\left(x_{0}\right)\right) \notin A_{0}\right) \mathcal{M}^{\varepsilon}\left(p_{i-1}\right) .
$$

We now want to estimate the probability appearing on the right-hand side by $\gamma$, which is indeed enough to infer (34). First let us transform the whole space by $M_{0}$ :

$$
Q_{1}:=\left(\mathrm{id}, M_{0}\right) Q_{0}, \quad A_{1}:=\left(\mathrm{id}, M_{0}\right) A_{0},
$$

and note that $\tau_{0}=\sup \left\{s<t_{0}:\left(s, M_{0} U_{t_{0}, s}\left(x_{0}\right)\right) \in A_{1} \cup \partial Q_{1}\right\}$.

Let us now apply Lemma 4.14 with the following choice of parameters:

- $p=p_{i}, r=r_{0}, c=2^{1 / 4} c_{0}+1$;

- $\left(\bar{B}_{t}\right)_{t \geq 0}=\left(B_{t_{0}-t}-B_{t_{0}}\right)_{t \geq 0},\left(\bar{\Omega},\left(\overline{\mathcal{F}}_{t}\right)_{t \geq 0}, \bar{P}\right)=\left(\hat{\Omega}, \sigma\left(\left(\bar{B}_{s}\right)_{s \in[0, t]}\right)_{t \geq 0}, \hat{P}\right)$;

- $A=\left\{(t, x):(-t, x) \in A_{1}-\left(t_{0}, M_{0} x^{\prime}\right)\right\}, n=n_{X_{\bar{t}}\left(x^{\prime}\right)}$;

- $(t, x)=\left(0, M_{0} x_{0}-M_{0} x^{\prime}\right)$;

- $\xi_{s}=M_{0} U_{t_{0}, t_{0}-s}\left(x_{0}\right)-M_{0} x_{0}$

$$
=\int_{0}^{s} M_{0} \beta_{t_{0}-s^{\prime}}\left(U_{t_{0}, t_{0}-s^{\prime}}\left(x_{0}\right)\right) d s^{\prime}+\int_{0}^{s} M_{0} \bar{\rho}_{t_{0}-s^{\prime}}\left(U_{t_{0}, t_{0}-s^{\prime}}\left(x_{0}\right)\right) d \bar{B}_{s^{\prime}} ;
$$

- $\delta=\kappa 2^{1 / 2}, \Delta=K 2^{1 / 2}$.

Let us verify the assumptions of Lemma 4.14. The measurability conditions are satisfied by construction. The bound on the drift is satisfied due to property (d) of $j_{0}$. Concerning the bounds on the diffusion, first note that as seen above, property (c) of $j_{0}$ implies that whenever $\left(s, U_{t_{0}, s}\left(x_{0}\right)\right) \in Q_{0},\left|z^{\prime}-\left(s, U_{t_{0}, s}\left(x_{0}\right)\right)\right| \leq \delta_{0}$, and so

$$
\begin{aligned}
M_{0} \bar{\rho}_{s}\left(U_{t_{0}, s}\left(x_{0}\right)\right) & =M_{0}\left(\nabla X_{s}\left(U_{t_{0}, s}\left(x_{0}\right)\right)\right)^{-1} \rho_{s}\left(X_{s}\left(U_{t_{0}, s}\left(x_{0}\right)\right)\right) \\
& =M\left(z^{\prime}\right) M^{-1}\left(\left(s, U_{t_{0}, s}\left(x_{0}\right)\right)\right) \rho_{s}\left(X_{s}\left(U_{t_{0}, s}\left(x_{0}\right)\right)\right),
\end{aligned}
$$

and the definition of $\delta_{0}$ along with the assumed bounds on $2 \bar{a}=\rho \rho^{*}$ implies the claimed bounds. The condition on $(t, x)$ is straightforward and follows from (35). 
As for the condition on $A$, first note that with denoting $\bar{x}:=X_{\bar{t}}\left(x^{\prime}\right) \in \partial G$,

$$
\begin{aligned}
\mathcal{R}: & =\left\{y:\left\langle y-M_{0} x^{\prime}, n\right\rangle \geq 2^{-p_{i} / 2-1}\right\} \cap B_{2 r_{0} 2^{-p_{i} / 2}}\left(M_{0} x^{\prime}\right) \\
& \subset\left\{y:\left\langle\frac{y-M_{0} x^{\prime}}{\left|y-M_{0} x^{\prime}\right|}, n\right\rangle \geq 1 /\left(4 r_{0}\right)\right\} \cap B_{2 r_{0} 2^{-p_{i} / 2}}\left(M_{0} x^{\prime}\right) \\
& =\left\{y:\left\langle\frac{y-M_{0} X_{\bar{t}}^{-1} \bar{x}}{\left|y-M_{0} X_{\bar{t}}^{-1} \bar{x}\right|}, n\right\rangle \geq 1 /\left(4 r_{0}\right)\right\} \cap B_{2 r_{0} 2^{-p_{i} / 2}}\left(M_{0} X_{\bar{t}}^{-1} \bar{x}\right) .
\end{aligned}
$$

Denoting further $\tilde{x}:=M_{0} X_{\bar{t}}^{-1} \bar{x}$, since one has $\nabla\left(X_{\bar{t}} M_{0}^{-1}\right)(\tilde{x})=I$, each $y$ in the latter set satisfies

$$
X_{\bar{t}} M_{0}^{-1} y-\bar{x}=y-\tilde{x}+e,
$$

where

$$
|e| \leq|y-\tilde{x}|^{2} \tilde{\mu} \leq|y-\tilde{x}| 2 r_{0} 2^{-p_{i} / 2} \tilde{\mu} \leq|y-\tilde{x}| /\left(32 r_{0}\right)
$$

by property (a) of $j_{0}$. In particular, a very crude application of this bound implies

$$
|y-\tilde{x}| / 2 \leq|y-\tilde{x}+e| \leq 2|y-\tilde{x}|,
$$

and hence

$$
\begin{aligned}
\left\langle\frac{X_{\bar{t}} M_{0}^{-1} y-\bar{x}}{\left|X_{\bar{t}} M_{0}^{-1} y-\bar{x}\right|}, n\right\rangle & \geq\left\langle\frac{y-\tilde{x}}{|y-\tilde{x}+e|}, n\right\rangle-\frac{|e|}{|y-\tilde{x}+e|} \\
& \geq \frac{1}{2}\left\langle\frac{y-\tilde{x}}{|y-\tilde{x}|}, n\right\rangle-\frac{1}{16 r_{0}} \geq \frac{1}{16 r_{0}} .
\end{aligned}
$$

Hence we can write

$$
\begin{aligned}
\mathcal{R} & \subset M_{0} X_{\bar{t}}^{-1}\left(\left\{y:\left\langle\frac{y-\bar{x}}{|y-\bar{x}|}, n\right\rangle \geq\left(1 / 16 r_{0}\right)\right\} \cap B_{\left.2 \bar{\mu} r_{0} 2^{-p_{i} / 2}(\bar{x})\right)}\right. \\
& \subset M_{0} X_{\bar{t}}^{-1}\left(G^{c}\right),
\end{aligned}
$$

where for the last inclusion we used property (b) of $j_{0}$. Let us now take an arbitrary

$$
y^{*} \in\left\{y:\left\langle y-M_{0} x^{\prime}, n\right\rangle \geq\left(2^{1 / 4} c_{0}+1 / 2\right) 2^{-p_{i} / 2}\right\} \cap B_{\left(r_{0}+1\right) 2^{-p_{i} / 2}}\left(M_{0} x^{\prime}\right)
$$

and an $s \in\left[t_{0}-2^{-p_{i}}, t_{0}\right]$. Denote $\bar{y}:=X_{s} M_{0}^{-1} y^{*}$ and $\tilde{y}:=M_{0} X_{\bar{t}}^{-1} \bar{y}$. Then one has

$$
\begin{aligned}
\tilde{y}-y^{*} & =M_{0}\left(X_{\bar{t}}^{-1} \bar{y}-X_{s}^{-1} \bar{y}\right) \\
& =M_{0} M^{-1}\left(\bar{t}, X_{\bar{t}}^{-1} \bar{y}\right) M\left(\bar{t}, X_{\bar{t}}^{-1} \bar{y}\right)\left(X_{\bar{t}}^{-1} \bar{y}-X_{s}^{-1} \bar{y}\right)
\end{aligned}
$$

We have

$$
\begin{aligned}
\left|X_{\bar{t}}^{-1} \bar{y}-x^{\prime}\right| & \leq\left|X_{\bar{t}}^{-1} X_{s} M_{0}^{-1} y^{*}-M_{0}^{-1} y^{*}\right|+\left|M_{0}^{-1} y^{*}-x^{\prime}\right| \\
& \leq \sup _{y \in \mathbb{R}^{d}}\left|X_{\bar{t}}^{-1} X_{s} y-y\right|+\bar{\mu} r_{0} 2^{-p_{i} / 2} \leq \delta_{0}
\end{aligned}
$$


by property (c) of $j_{0}$. Hence, using the defining property (31) of $p_{i}$, from (36) we get

$$
\left|\tilde{y}-y^{*}\right| \leq 2^{1 / 4} c_{0} 2^{-p_{i} / 2} .
$$

It follows that $\tilde{y} \in \mathcal{R} \subset M_{0} X_{\bar{t}}^{-1}\left(G^{c}\right)=M_{0} \tilde{Q}_{\bar{t}}^{c}$, and so

$$
y^{*}=M_{0} X_{s}^{-1} X_{\bar{t}} M_{0}^{-1} \tilde{y} \in M_{0} \tilde{Q}_{s}^{c} .
$$

Hence

$$
\begin{aligned}
& {\left[t_{0}-2^{-p_{i}}, t_{0}\right]\left(\left\{y:\left\langle y-M_{0} x^{\prime}, n\right\rangle \geq\left(2^{1 / 4} c_{0}+1 / 2\right) 2^{-p_{i} / 2}\right\} \cap B_{\left(r_{0}+1\right) 2^{-p_{i} / 2}}\left(M_{0} x^{\prime}\right)\right)} \\
& \quad \subset\left(\mathrm{id}, M_{0}\right) \tilde{Q}^{c} .
\end{aligned}
$$

Let now $j_{1}=j_{1}(\varepsilon, \omega)$ be the largest integer such that the Hausdorff distance between $\tilde{Q}$ and $\tilde{Q}^{\varepsilon}$ is smaller than $2^{-j_{1}-1}$. Then if $p_{i} \leq j_{1}$, we get

$$
\begin{aligned}
& {\left[t_{0}-2^{-p_{i}}, t_{0}\right]\left(\left\{y:\left\langle y-M_{0} x^{\prime}, n\right\rangle \geq\left(2^{1 / 4} c_{0}+1\right) 2^{-p_{i} / 2}\right\} \cap B_{r_{0} 2^{-p_{i} / 2}}\left(M_{0} x^{\prime}\right)\right)} \\
& \quad \subset\left(\mathrm{id}, M_{0}\right)\left(Q_{0} \backslash \tilde{Q}^{\varepsilon}\right) .
\end{aligned}
$$

By a simple translation and reflection, we get the desired property of $A$. Also notice that $\tau_{t, x}=t_{0}-\tau_{0}$ and

$$
\left\{\left(\tau_{0}, U_{t_{0}, \tau_{0}}\left(x_{0}\right)\right) \notin A_{0}\right\}=\left\{\left(\tau_{0}, M_{0} U_{t_{0}, \tau_{0}}\left(x_{0}\right)\right) \notin A_{1}\right\}=\left\{\left(\tau_{t, x}, x+\xi_{\tau_{t, x}}\right) \notin A\right\} .
$$

Applying Lemma 4.14 therefore yields

$$
\hat{P}\left(\left(\tau_{0}, U_{t_{0}, \tau_{0}}\left(x_{0}\right)\right) \notin A_{0}\right) \leq \gamma
$$

as claimed and the proof is concluded.

\section{APPENDIX}

The following lemma is a variation on Kolmogorov's Hölder-estimate, with the difference being that the two-parameter family we estimate here is not necessarily represented as increments of a (one-parameter) function.

LEMMA A.15. Let $(V,|\cdot|)$ be a normed vector space and let $\left(D_{s, t}\right)_{s, t \in[0, T]}$ and $\left(E_{s, s^{\prime}, t, t^{\prime}}\right)_{s, s^{\prime}, t, t^{\prime} \in[0, T]}$ be two families of $V$-valued random variables, satisfying

$$
\begin{aligned}
\left|D_{s, t}\right| & \leq\left|D_{s, r}\right|+\left|D_{r, t}\right|+\left|E_{s, r, r, t}\right|, \\
\left|E_{s_{1}, s_{2}, s_{3}, s_{4}}\right| & \leq\left(\left|E_{s_{1}, t, s_{3}, s_{4}}\right|+\left|E_{t, s_{2}, s_{3}, s_{4}}\right|\right) \wedge\left(\left|E_{s_{1}, s_{2}, s_{3}, t}\right|+\left|E_{t, s_{2}, t, s_{4}}\right|\right)
\end{aligned}
$$

for all choice of arguments. Suppose furthermore that $D$ is almost surely continuous in both arguments and that for some $p \geq 1, C>0, \alpha, \alpha_{1}, \alpha_{2}>0$ the bounds

$$
\begin{aligned}
\mathbb{E}\left|D_{s, t}\right|^{p} & \leq C|s-t|^{\alpha}, \\
\mathbb{E}\left|E_{s, s^{\prime}, t, t^{\prime}}\right|^{p} & \leq C\left|s-s^{\prime}\right|^{\alpha_{1}}\left|t-t^{\prime}\right|^{\alpha_{2}}
\end{aligned}
$$


hold uniformly in $s, s^{\prime}, t, t^{\prime}$. Then, if $0<p \gamma<(\alpha-1) \wedge\left(\alpha_{1}+\alpha_{2}-2\right)$, then

$$
\mathbb{E}\left(\sup _{s \neq t \in[0, T]}|s-t|^{-\gamma}\left|D_{s, t}\right|\right)^{p} \leq C N\left(T, \gamma, \alpha, \alpha_{1}, \alpha_{2}, p\right) .
$$

ProOF. We assume without loss of generality $T=1$. Introduce the notation $\mathcal{D}_{k}=2^{-k} \mathbb{Z} \cap[0,1]$ and $\mathcal{D}=\bigcup_{k=0}^{\infty} \mathcal{D}_{k}$ for the dyadic numbers and note that due to the continuity of $D$, it suffices to take supremum over $s, t \in \mathcal{D}$ in (39). For fixed $s, t \in \mathcal{D}$, let $n \in \mathbb{N}$ be such that $2^{-n-1} \leq|s-t| \leq 2^{-n}$. Let $\left(s_{k}\right)_{k \geq n}$ and $\left(t_{k}\right)_{k \geq n}$ be two sequences such that $s_{k}, t_{k} \in \mathcal{D}_{k},\left|s_{n}-t_{n}\right| \leq 2^{-n},\left|s_{k+1}-s_{k}\right| \vee\left|t_{k+1}-t_{k}\right| \leq$ $2^{k+1}$, and that for some large enough $N,\left|s_{k}-s\right| \vee\left|t_{k}-t\right|=0$ for all $k \geq N$. One then has, due to (37),

$$
\begin{aligned}
\left|D_{s, t}\right| \leq & \left|D_{s, s_{n}}\right|+\left|D_{s_{n}, t_{n}}\right|+\left|D_{t_{n}, t}\right|+\left|E_{s, s_{n}, s_{n}, t}\right|+\left|E_{S_{n}, t_{n}, t_{n}, t}\right| \\
\leq & \sum_{k=n}^{N}\left|D_{s_{k+1}, s_{k}}\right|+\sum_{k=n}^{N}\left|E_{s, s_{k+1}, s_{k+1}, s_{k}}\right|+\left|D_{s_{n}, t_{n}}\right| \\
& +\sum_{k=n}^{N}\left|D_{t_{k}, t_{k+1}}\right|+\sum_{k=n}^{N}\left|E_{t_{k}, t_{k+1}, t_{k+1}, t}\right|+\left|E_{s, s_{n}, s_{n}, t}\right|+\left|E_{s_{n}, t_{n}, t_{n}, t}\right| \\
= & : \sum_{i=1}^{7} I_{i} .
\end{aligned}
$$

Clearly, each of $I_{1}, I_{3}$ and $I_{4}$ is bounded (up to a universal constant) by

$$
2^{-\gamma n} \sup _{k \geq 0} \sup _{r \in \mathcal{D}_{k}}\left|D_{r, r+2^{-k}}\right| 2^{\gamma k}=: 2^{-\gamma n} A .
$$

Choose $\gamma_{1}, \gamma_{2}>0$ such that $\gamma_{1}+\gamma_{2}=\gamma$ and $p \gamma_{i}<\alpha_{i}-1$ for $i=1,2$. Then each of $I_{2}, I_{5}, I_{6}$ and $I_{7}$ is bounded (up to a universal constant) by

$$
2^{-\gamma n} \sup _{k, k^{\prime} \geq 0} \sup _{\substack{r \in \mathcal{D}_{k} \\ r^{\prime} \in \mathcal{D}_{k^{\prime}}}}\left|E_{r, r+2^{-k}, r^{\prime}, r^{\prime}+2^{-k^{\prime}}}\right| 2^{\gamma_{1} k} 2^{\gamma_{2} k^{\prime}}=: 2^{-\gamma n} B .
$$

This can be easily seen, for example, in the case of $I_{2}$ (the other terms can be treated similarly), from

$$
I_{2} \leq \sum_{k=n}^{N} \sum_{k^{\prime}=k+1}^{N}\left|E_{s_{k^{\prime}+1}, s_{k^{\prime}}, s_{k+1}, s_{k}}\right| \leq B \sum_{k=n}^{\infty} \sum_{k^{\prime}=k+1}^{\infty} 2^{-k^{\prime} \gamma_{1}} 2^{-k \gamma_{2}} \leq B 2^{-n\left(\gamma_{1}+\gamma_{2}\right)}
$$

Therefore,

$$
\mathbb{E}\left(\sup _{s \neq t \in[0, T]}|s-t|^{-\gamma}\left|D_{s, t}\right|\right)^{p} \leq 7^{p} \mathbb{E}(A \vee B)^{p},
$$


and it remains to bound $\mathbb{E}(A \vee B)^{p}$. Using the bounds (38) and the conditions on the exponents, one has, up to constants depending on $p$ and the exponents,

$$
\begin{aligned}
\mathbb{E}(A \vee B)^{p} \leq & \mathbb{E} \sup _{k, k^{\prime} \geq 0} \sup _{\substack{r \in \mathcal{D}_{k} \\
r^{\prime} \in \mathcal{D}_{k^{\prime}}}}\left|D_{r, r+2^{-k}}\right|^{p} 2^{\gamma k p}+\left|E_{r, r+2^{-k}, r^{\prime}, r^{\prime}+2^{-k^{\prime}}}\right|^{p} 2^{\gamma_{1} k p} 2^{\gamma_{2} k^{\prime} p} \\
\leq & \sum_{k \geq 0} \sum_{r \in \mathcal{D}_{k}} \mathbb{E}\left|D_{r, r+2^{-k}}\right|^{p} 2^{\gamma k p} \\
& +\sum_{k, k^{\prime} \geq 0} \sum_{\substack{r \in \mathcal{D}_{k} \\
r^{\prime} \in \mathcal{D}_{k^{\prime}}}} \mathbb{E}\left|E_{r, r+2^{-k}, r^{\prime}, r^{\prime}+2^{-k^{\prime}}}\right|^{p} 2^{\gamma_{1} k p} 2^{\gamma_{2} k^{\prime} p} \\
\leq & C \sum_{k \geq 0} 2^{k} 2^{-\alpha k} 2^{\gamma k p}+C \sum_{k, k^{\prime} \geq 0} 2^{k+k^{\prime}} 2^{-\alpha_{1} k} 2^{-\alpha_{2} k^{\prime}} 2^{\gamma_{1} k p} 2^{\gamma_{2} k^{\prime} p} \leq C .
\end{aligned}
$$

\section{REFERENCES}

[1] Dareiotis, K. and Gerencsér, M. (2015). On the boundedness of solutions of SPDEs. Stoch. Partial Differ. Equ., Anal. Computat. 3 84-102. MR3312593

[2] DAREiotis, K. A. and GYÖNGY, I. (2014). A comparison principle for stochastic integrodifferential equations. Potential Anal. 41 1203-1222. MR3269720

[3] Flandoli, F. (1990). Dirichlet boundary value problem for stochastic parabolic equations: Compatibility relations and regularity of solutions. Stochastics Stochastics Rep. 29 331357. MR1042066

[4] Friz, P. K. and HAIRER, M. (2014). A Course on Rough Paths: With an Introduction to Regularity Structures. Springer, Cham. MR3289027

[5] Gerencsér, M. and GYöngY, I. (2018). A Feynman-Kac formula for stochastic Dirichlet problems. Stochastic Process. Appl. 129 995-1012.

[6] Gerencsér, M., Gyöngy, I. and Krylov, N. (2015). On the solvability of degenerate stochastic partial differential equations in Sobolev spaces. Stoch. Partial Differ. Equ. Anal. Comput. 3 52-83. MR3312592

[7] KIM, K.-H. (2004). On $L_{p}$-theory of stochastic partial differential equations of divergence form in $C^{1}$ domains. Probab. Theory Related Fields 130 473-492. MR2102888

[8] KIM, K.-H. (2004). On stochastic partial differential equations with variable coefficients in $C^{1}$ domains. Stochastic Process. Appl. 112 261-283. MR2073414

[9] KrYLov, N. (2008). A brief overview of the $L_{p}$-theory of SPDEs. Theory Stoch. Process. 14 71-78. MR2479735

[10] KRYLOV, N. V. (1994). A $W_{2}^{n}$-theory of the Dirichlet problem for SPDEs in general smooth domains. Probab. Theory Related Fields 98 389-421. MR1262972

[11] Krylov, N. V. (2001). Some properties of traces for stochastic and deterministic parabolic weighted Sobolev spaces. J. Funct. Anal. 183 1-41. MR1837532

[12] Krylov, N. V. (2002). Introduction to the Theory of Random Processes. Graduate Studies in Mathematics 43. Amer. Math. Soc., Providence, RI. MR1885884

[13] KRYLOV, N. V. (2003). Brownian trajectory is a regular lateral boundary for the heat equation. SIAM J. Math. Anal. 34 1167-1182. MR2001664

[14] KRYLOV, N. V. (2003). One more square root law for Brownian motion and its application to SPDEs. Probab. Theory Related Fields 127 496-512. MR2021193

[15] Krylov, N. V. (2011). On the Itô-Wentzell formula for distribution-valued processes and related topics. Probab. Theory Related Fields 150 295-319. MR2800911 
[16] Krylov, N. V. and Lototsky, S. V. (1999). A Sobolev space theory of SPDEs with constant coefficients on a half line. SIAM J. Math. Anal. 30 298-325. MR1664761

[17] Krylov, N. V. and Lototsky, S. V. (1999). A Sobolev space theory of SPDEs with constant coefficients in a half space. SIAM J. Math. Anal. 31 19-33. MR1720129

[18] Krylov, N. V. and RozovskiĬ, B. L. (1981). Stochastic evolution equations. J. Sov. Math. 16 1233-1277. MR0570795

[19] KunitA, H. (1984). Stochastic differential equations and stochastic flows of diffeomorphisms. In École D'été de Probabilités de Saint-Flour, XII-1982. Lecture Notes in Math. 1097 143-303. Springer, Berlin. MR0876080

[20] Kunita, H. (1997). Stochastic Flows and Stochastic Differential Equations. Cambridge Studies in Advanced Mathematics 24. Cambridge Univ. Press, Cambridge. Reprint of the 1990 original. MR1472487

[21] Lототs KY, S. V. (1999). Dirichlet problem for stochastic parabolic equations in smooth domains. Stochastics Stochastics Rep. 68 145-175. MR1742721

[22] LототsKy, S. V. (2000). Sobolev spaces with weights in domains and boundary value problems for degenerate elliptic equations. Methods Appl. Anal. 7 195-204. MR1796011

[23] LототsкY, S. V. (2001). Linear stochastic parabolic equations, degenerating on the boundary of a domain. Electron. J. Probab. 6 Art. ID 24. MR1873301

[24] PARdoux, E. (1975). Équations aux dérivées partielles stochastiques de type monotone. Etude de solutions fortes de type Itô. Ph.D. thesis, Univ. Paris-Sud. MR0651582

[25] Revuz, D. and Yor, M. (2004). Continuous Martingales and Brownian Motion, 3rd ed. Grundlehren der Mathematischen Wissenschaften [Fundamental Principles of Mathematical Sciences] 293. Springer, Berlin. MR 1083357

[26] Triebel, H. (1995). Interpolation Theory, Function Spaces, Differential Operators, 2nd ed. Johann Ambrosius Barth, Heidelberg. MR1328645

IST AUSTRIA

AM CAMPUS 1

3400, KLOSTERNEUBURG

AUSTRIA

E-MAIL: mate.gerencser@ist.ac.at 